\title{
The performance of externally managed REITs in Asia
}

Article

Accepted Version

Chong, W. L., Ting, K. H. and Cheng, F. F. (2017) The performance of externally managed REITs in Asia. Journal of Property Investment \& Finance, 35 (2). pp. 200-227. ISSN 1463-578X doi: https://doi.org/10.1108/JPIF-08-2016-0067 Available at https://centaur.reading.ac.uk/69795/

It is advisable to refer to the publisher's version if you intend to cite from the work. See Guidance on citing.

To link to this article DOI: http://dx.doi.org/10.1108/JPIF-08-2016-0067

Publisher: Emerald Group Publishing Limited

All outputs in CentAUR are protected by Intellectual Property Rights law, including copyright law. Copyright and IPR is retained by the creators or other copyright holders. Terms and conditions for use of this material are defined in the End User Agreement.

\section{www.reading.ac.uk/centaur}

\section{CentAUR}

Central Archive at the University of Reading

Reading's research outputs online 


\title{
The Performance of Externally Managed REITs in Asia: Further Evidence from Free Cash Flow and Agency Cost
}

\author{
Wei Lan Chong, Universiti Putra Malaysia* \\ Kien Hwa Ting, Universiti Teknologi MARA, Malaysia** \\ Fan Fah Cheng, Universiti Putra Malaysia*** \\ *Putra Business School, Universiti Putra Malaysia, 43400 UPM Serdang, Selangor, Malaysia. Tel: 03-89467679 Fax: 03-8943
4019 Email: eve_chong@email.com
** Centre for Real Estate Research, Faculty of Architecture, Planning \& Surveying, Universiti Teknologi MARA (UiTM), 40450
Shah Alam, Selangor, Malaysia. Tel: 03-5544 4217 Fax: 03-5544 4353 Email: tingkien@salam.uitm.edu.my
*** Department of Accounting and Finance, Faculty of Economics and Management, Universiti Putra Malaysia, 43400 UPM
Serdang, Selangor, Malaysia. Tel: 03-89467679 Fax: 03-89466188
}

\section{ABSTRACT}

This paper examines the impact of free cash flow on the agency costs and how these free cash flow and agency costs affect the performance of REITs in Asia. Unlike previous studies that focus on conventional non-regulated industry, the Asian REIT industry being a highly regulated industry provides a new context for further research.

The results indicate that free cash flow and agency costs persist over time in Asian REITs even though REITs are in a highly regulated industry. The findings also imply that REIT managers face substantial costs when they wish to adjust to the equilibrium level of agency costs, whereby the optimum level is always dynamic and not constant over time and moves with the changes in the determinants of agency costs. These agency costs persist over time and have significant impacts on the performance of REITs in Asia.

Key words: free cash flow, agency costs, performance, dynamic, Asian REITs, GMM method.

\subsection{INTRODUCTION}

Since its introduction in 2002, Asian REITs have been booming and growing significantly, especially after post-Global Financial Crisis. Until today, REIT markets are now established in many countries in Asia Pacific region including Japan, Singapore and Hong Kong and there are 246 REITs in Asia Pacific region with a total market capitalization of over US $\$ 270$ billion by December 2015 which accounts for $18 \%$ of the global REIT market (Bloomberg). The new entry REIT country includes Pakistan.

Although there are a lot of research on agency conflicts such as free cash flow and agency costs but these studies are on the conventional public companies. These researches broadly cover the aspects of free cash flow and the relevant free cash flow issues such as dividend policy, agency costs etc.; but only focus on non-regulated and conventional listed companies.

For REITs, there are limited empirical researches on these aspects and most of these studies are US-centric. There are some empirical studies such as documented in Chen, Wang and Shyu (2012) examine how deviations from expected optimal cash holdings affect future stock returns in the US REIT industry, the combined 
impact of corporate governance and excess cash holdings on the propensity of firms to become bidders (Ghosh, Petrova, and Xiao, 2012), institutional investors and firm efficiency of REITs (Chung, Fung, and Hung, 2012).

There is no research focusing on Asian REITs that examine the issues of free cash flow and agency costs. The primary objective of this research is to examine the impacts of free cash flow on agency costs. In addition, this paper also assesses the impacts of these free cash flows and agency costs on the performance, value and returns of REITs in Asia even though REITs are in a highly regulated industry. Unlike previous studies that focus on conventional non-regulated industry, the Asian REIT industry being a highly regulated industry provides a new context for further research.

The panel data of the four biggest market capitalization of Asian REITs, namely, Japan, Singapore, Hong Kong and Malaysia is being used and the study period is from year 2002 to 2012. This study employs GMM method which is more robust compared to previous studies in conventional non-regulated industry that used pooled OLS method and panel data method. The Sargan test, $A R(1)$ and $A R(2)$ tests are being used to assess the models.

The findings drawn from this study can be used as a useful information and reference for all levels of the REIT industry in Asia.

\subsection{LITERATURE REVIEW}

\subsection{Free Cash Flow}

The free cash flow (FCF) theory was originally developed by Jensen (1986) in which free cash flow is defined as net cash flows after deducting the needs of positive net present value (NPV) projects. Many research studies on free cash flow are of nonREITs such as in Gul and Tsui (1997), Brush, Philip and Margaretha (2000), Gul and Tsui (2001), Gregory (2005), Richardson (2006), Chang, Chen, Hsing and Huang (2007), Oprea (2008), Chen, Chou and Chou (2009), and Wang (2010).

For REITs, the results in Chen et al. (2012) show that cash holdings are inversely related to leverage and credit line access. This helps to alleviate the agency problem of free cash flow (Jensen, 1986). Their results indicate that the cash holding is positively associated with growth opportunities. The REIT managers access future growth opportunities and the external cost of capital, hold more cash for future investment opportunities, thus, this supports the pecking order theory. These findings are similar to those of previous studies (Myers and Majluf, 1984; Jensen, 1986; Kim, Mauer and Sherman, 1998; Opler, Pinkowitz, Stulz and Williamson, 1999; Hardin III, Highfield, Hill and Kelly, 2009).

In addition, Chen et al. (2012) also attribute that REIT firms that do not hold optimal cash holdings, the stock returns diminish. This is consistent with the findings of $\mathrm{Li}$ (2008) and Oler and Picconi (2010). Nevertheless, according to Chen et al. (2012), the detrimental effects of deviations above the estimated optimal cash levels are insignificantly stronger than those of deviations below the optimal level. 
This also infers that the agency problems inherent in free cash flow (Jensen, 1986), the abandonment of investment opportunities (Myers et al., 1984), and the likelihood of bankruptcy will decrease REIT firms' stock returns. The divergence from the expected optimal cash holding further worsen the price performance, whether because of excess or insufficient cash.

Nevertheless, according to Chen et al. (2012), their results may suffer from sampling bias, specification bias, and estimation bias, thereby altering the estimated consequences.

The findings of Ghosh et al. (2012) reveal that REITs with higher excess cash and lower insider ownership have the tendency to become bidders. Their results also document that there is a significantly positive valuation effect associated with the excess cash holdings for firms with lower insider ownership and cumulative abnormal return surrounding the merger announcement. They attribute it to be consistent with the hypothesis that investors are not averse by the REIT managers' spending and expenditure on intra-industry acquisition.

\subsection{Agency Cost}

Many research studies on agency costs are on non-REITs such as studies done by Ang, Cole and Lin (2000), Singh and Davidson III (2003), Fleming, Heaney and McCosker (2005), Florackis (2008), McKnight and Weir (2009), Florackis and Ozkan (2009), Jelinek and Stuerke (2009), Chi and Lee (2010), Henry (2010), Wang (2010), Hijazi and Conover (2011), He (2012) and Iskandar, Bukit and Sanusi (2012).

For REITs, the findings of Chou, Hardin III, Hill and Kelly (2013) suggest that total, mandatory and discretionary dividends are valued by investors. Furthermore, the results also indicate that dividends are more highly valued for REITs with less transparent operating structure such as in UPREITs and REITs with more than fifty percent of its board comprised of insiders and affiliates. These findings are consistent with agency costs theory and imply that the discretionary dividends payout other than mandatory dividend payout is a substitute for weak corporate governance. The results are consistent with Ghosh and Sirmans (2006) and Feng, Ghosh and Sirmans (2007), which show that REITs substitute dividends for weaker governance.

Further, their results also suggest that cash flow generation (FFO) is an important determinant of value, and disbursement of this cash flow in the form of discretionary dividends can be value enhancing.

The results also imply that the market recognizes these discretionary dividends paid are more highly valued for firms with less transparent operating and governance structures (Chou et al., 2013). According to Chou et al. (2013), the market's recognition of this substitution should be relevant to REITs because of their dependence on external debt and equity markets. The willingness and ability to trade off forms of governance and policies reducing agency costs highlights the complexity of the interaction between firms and financial market participants. 
As a whole, there is limited research on REIT issues in Asia; whereas in US, there are more research studies and findings on US REITs. Perhaps in Asia, the development of Asian REITs is still in infancy stage as compare to the more mature market in US REITs.

Therefore, it is important to study the agency issues pertaining to the effects of free cash flow and agency costs on the performance of Asian REITs. This empirical study provides considerable insight into the impacts of free cash flows on the individual agency cost; and how free cash flow and the individual agency cost could have an influence on the performance, value and returns of Asian REITs.

\subsection{RESEARCH METHODOLOGY}

\subsection{Research Method}

The GMM Method is being used in this research study because firstly, the regressors in the model are endogenous. These regressors may be correlated with the error term and this could lead to biased coefficients. Thus, GMM method is required.

Secondly, in the random effects model, GLS estimator is biased in static model if these regressors are correlated with the error term.

Thirdly, there is time invariant firms' specific effect in the regression model. Although fixed effect model can be used, but the within estimator is biased and inconsistent as the number of individual increases and $\mathrm{T}$ is still relatively small. and $\mathrm{T}<10$.

Fourthly, the panel data in the study have a long firm dimension where $N>100$

Fifthly, the GMM method allows for empirical modelling of dynamic while accounting for individual-specific dynamics.

Moreover, following the studies in Wintoki, Linck and Netter (2012) and Flannery and Hankins (2013), the dynamic endogeneity, simultaneity and unobserved heterogeneity exist in the above regression models in the study. Thus, the GMM is more robust to be employed in this study.

\subsection{Data Collection}

The samples for this study comprise REITs from four major Asian REIT countries i.e. Japan, Singapore, Hong Kong and Malaysia. These countries are the 
leaders in Asian REIT markets which account for $94 \%$ of the total market capitalization of REITs in Asia (Newell, 2012).

The study period is from the commencement of REITs in each country which covers the period from 2002 to 2012. The introduction of REITs differs among the Asian countries i.e. 2001 in Japan, 2002 in Singapore; 2004 in Malaysia, and 2005 in Hong Kong. Hence, the data collected will be an unbalanced panel data. The REIT IPOs in 2012 are not included in this study.

These data and figures are collected from published annual company reports, company websites, REIT related publications, Thomson One Banker and Datastream.

\subsection{The Direct Agency Costs Regression Models}

\subsubsection{Free Cash Flow}

The free cash flow is employed and being used as proxy for free cash flow and perceived likelihood of agency problems (Lehn and Poulsen, 1989; Wu, 2004; Chi and Lee, 2010). The first measure of free cash flow 1 (FCF1) is defined as follows:

$F C F 1_{t}=\frac{O C F_{t}-\mathrm{Tax}_{\mathrm{t}}-\mathrm{IExp}_{\mathrm{t}}-\mathrm{CDiv}_{\mathrm{t}}-\mathrm{PDiv}_{\mathrm{t}}}{\text { BV Assets }}$

where

$\mathrm{OCF}_{\mathrm{t}}$ denotes operating cash flows at time $\mathrm{t}$;

Taxt denotes corporate income tax expense at time t;

IExpt denotes interest expense at time t;

CDivt denotes common stock dividends at time $t$;

PDivt denotes preferred stock dividends at time t;

BV Assetst denotes book value of assets at time $t$.

Following Chae, Kim and Lee (2009), the proxy for free cash flow 2 (FCF2) is defined as follows:

$F C F 2 t=\frac{\text { EBITDA }_{t}}{\text { BV Assets }_{t}}$

where

EBITDA $_{t}$ denotes earning before interests, taxes, depreciation, and amortization at time t;

BV Assett denotes book value of assets at time $t$.

In addition, following Lang, Stulz and Walking (1991) and Hijazi et al. (2011), the third alternative proxy for free cash flow 3 (FCF3) is as follows:

$\mathrm{FCF}_{\mathrm{t}}=\mathrm{FCFFOA}_{\mathrm{t}}-\mathrm{CE}_{\mathrm{t}}$ 
where

FCFFOA denotes free cash flow from operating activities at time t;

$\mathrm{CE}_{\mathrm{t}}$ denotes capital expenditure at time $\mathrm{t}$.

\subsubsection{Direct Agency Costs}

\subsubsection{Asset Turnover}

Following Ang et al. (2000), Singh et al. (2003), McKnight et al. (2009), Fleming et al. (2005), Florackis (2008), Florackis et al.(2009), Jelinek et al. (2009), Wang (2010), Hijazi et al. (2011) and Iskandar et al. (2012), there are few proxies for direct agency costs. The first proxy for direct agency costs is asset turnover which is the efficiency ratio of how REIT manager deploys its assets. The asset turnover is also defined as asset utilization ratio. The higher the sales-to-assets ratio indicates that the assets are generating significant sales and therefore, depicts that the lower is the agency costs.

On the contrary, lower sales-to-assets ratio suggests that there is inefficiency in REIT management in implementing its policies such as poor investment decision, excessive perquisite consumption. Therefore, the sales-to-assets ratio is inversely related to the agency costs. The asset turnover is defined as follows:

Ass $_{t}=\frac{\text { Sales }_{t}}{\text { Asset }_{t}}$

where

Ass $T_{t}$ denotes total asset turnover at time $t$;

Salest denotes net sales at time t;

Assetst denotes total assets at time t.

\subsubsection{Operating Expense Ratio}

The second direct agency costs proxy is the operating expense ratio. It is defined as operating expenses divided by net sales (Ang et al, 2000; Fleming et al., 2005; Jelinek et al., 2009; Wang, 2010; Hijazi et al., 2011; and Iskandar, et al., 2012). The operating expense ratio is as follows:

$\mathrm{OpeR}_{\mathrm{t}}=\frac{\mathrm{OpeE}_{\mathrm{t}}}{\text { Sales }_{\mathrm{t}}}$

where

Ope $R_{t}$ denotes operating expense ratio at time $t$;

Ope $E_{t}$ denotes operating expenses at time $t$;

Salest denotes net sales at time $t$. 


\subsubsection{Selling, General and Administrative Expenses Ratio}

The third direct agency costs proxy is selling, general and administrative expenses ratio (SGAR). It is defined as selling, general and administrative expenses scaled by net sales (Singh et al., 2003; Florackis, 2008; Florackis et al., 2009; Hijazi et al., 2011).

The SGA expense is defined as the costs related to REIT management function, and costs to the sales. For instances, managerial salaries, rent, insurance, utilities, supplies, and advertising costs. Higher SGA expenses may induce higher agency costs, thus, this reflects the managerial discretionary expenses and excessive perquisite consumption. The SGAR is defined as follows:

$\mathrm{SGAR}_{\mathrm{t}}=\frac{\mathrm{SGA}_{\mathrm{t}}}{\text { Sales }_{\mathrm{t}}}$

where

SGARt denotes selling, general and administrative expenses ratio at time t; $S G A_{t}$ denotes selling, general and administrative expenses at time $t$;

Salest denotes net sales at time t.

\subsubsection{Net Operating Income Volatility}

The fourth direct agency costs proxy is net operating income volatility. It is defined as the standard deviation of net operating income scaled by total sales over the sample period (Wang, 2010). The NOIVol1 is defined as follows:

NOIVol $1 \mathrm{t}=$ STD of $\left(\frac{\mathrm{NOI}_{\mathrm{t}}}{\text { Sales }_{\mathrm{t}}}\right)$

where

NOIVol $1_{t}$ denotes net operating income volatility 1 at time $t$;

NOlt denotes net operating income at time t;

STD denotes standard deviation;

Salest denotes total sales at time $t$.

\subsubsection{Net Income Volatility}

The fifth direct agency costs proxy is net income volatility (Wang, 2010). It is defined as the standard deviation of net income scaled by total sales over the sample period. The NIVol1 is defined as follows: 
NIVol $1_{t}=$ STD of $\left(\frac{\mathrm{NI}_{\mathrm{t}}}{\text { Sales }_{\mathrm{t}}}\right)$

where

NIVol $1_{t}$ denotes net income volatility 1 at time $t ;$

$\mathrm{Nl}_{\mathrm{t}}$ denotes net income at time $\mathrm{t}$;

STD denotes standard deviation;

Salest denotes total sales at time t.

However, the firm's individual and specific effects may also affect the free cash flow and direct agency costs proxies. Hence, this may cause heteroscedasticity in the model. Therefore, control variables are being used in order to control for firm's individual and specific effect in the model.

The direct agency costs regression models are as follows:

AssT $_{t}=\beta_{0}+\beta_{1}$ FCF $_{t}+\beta_{2}$ Size $_{t}+\beta_{3}$ Age $_{t}+\beta_{4}$ Growth $_{t}+\beta_{5}$ Leverage $_{t}+\beta_{6}$ DivPayt $_{t}$

$+\beta_{7}$ Profitabilityt $+\varepsilon t$

Ope Rt $_{t}=\beta_{0}+\beta_{1} \mathrm{FCF}_{t}+\beta_{2}$ Size $_{t}+\beta_{3}$ Age $_{t}+\beta_{4}$ Growth $_{t}+\beta_{5}$ Leverage $_{t}+\beta_{6}$ DivPay $_{t}$

$+\beta_{7}$ Profitability $+\varepsilon_{t}$

SGAR $_{t}=\beta_{0}+\beta_{1}$ FCF $_{t}+\beta_{2}$ Size $_{t}+\beta_{3}$ Age $_{t}+\beta_{4}$ Growth $_{t}+\beta_{5}$ Leverage $_{t}+\beta_{6}$ DivPayt $_{t}$ $+\beta 7$ Profitability $+\varepsilon t$

NOIVol1 $_{t}=\beta_{0}+\beta_{1}$ FCF $_{t}+\beta_{2}$ Size $_{t}+\beta_{3}$ Age $_{t}+\beta_{4}$ Growth $_{t}+\beta_{5}$ Leverage $_{t}+\beta_{6}$ DivPay $_{t}$ $+\beta_{7}$ Profitability $_{t}+\varepsilon t$

NIVol1 $_{t}=\beta_{0}+\beta_{1}$ FCF $_{t}+\beta_{2}$ Size $_{t}+\beta_{3}$ Age $_{t}+\beta_{4}$ Growth $_{t}+\beta_{5}$ Leverage $_{t}+\beta_{6}$ DivPay $_{t}$ $+\beta_{7}$ Profitability $+\varepsilon_{t}$

\subsection{The REIT Performance Regression Model}

The return on assets (ROA) and return on equity (ROE) are being used to measure the performance of REITs in Asia. as follows:

The general regression models of REIT agency costs on REIT performance are

$\mathrm{ROA}_{t}=\beta_{0}+\beta_{1} \mathrm{FCF}_{t}+\beta_{2} \mathrm{AssT}_{t}+\beta_{3} \mathrm{OpeR}_{t}+\beta_{4} \mathrm{SGAR}_{t}+\beta_{5} \mathrm{NOIVol}_{t}+\beta_{6} \mathrm{NIVol}_{t}$

$+\beta_{7}$ Size $_{t}+\beta_{8}$ Age $_{t}+\beta_{9}$ Growth $_{t}+\beta_{10}$ Leverage $_{t}+\beta_{11}$ DivPay $_{t}+\beta_{12}$ Profitability $_{t}+\varepsilon_{t}$

ROE $_{t}=\beta_{0}+\beta_{1} \mathrm{FCF}_{t}+\beta_{2} \mathrm{AssT}_{t}+\beta_{3} \mathrm{OpeR}_{t}+\beta_{4} \mathrm{SGAR}_{t}+\beta_{5} \mathrm{NOIVol}_{t}+\beta_{6} \mathrm{NIVol}_{t}$

$+\beta_{7}$ Size $_{t}+\beta_{8}$ Age $_{t}+\beta_{9}$ Growth $_{t}+\beta_{10}$ Leverage $_{t}+\beta_{11}$ DivPay $_{t}+\beta_{12}$ Profitability $_{t}+\varepsilon t$ 


\subsection{The REIT Value Regression Model}

\subsubsection{REIT Value}

The Tobin $q$ is defined as follows:

Tobin $\mathrm{q}_{\mathrm{t}}=\frac{\mathrm{MVA}_{\mathrm{t}}+\mathrm{PS}_{\mathrm{t}}+\mathrm{Debt}_{\mathrm{t}}}{\text { Book Value of Asset }}$

where

$\mathrm{MVA}_{t}$ denotes market value of common equity at time $t$;

$P S_{t}$ denotes market value of preferred equity at time $t$;

Debt denotes book value of total debt at time t;

Book Value of Assett denotes book value of total assets at time $t$. follows:

The general regression model of REIT agency costs on REIT value is as

Tobin $q_{t}=\beta_{0}+\beta_{1} \mathrm{FCF}_{t}+\beta_{2} \mathrm{AssT}_{t}+\beta_{3} \mathrm{OpeR}_{t}+\beta_{4} \mathrm{SGAR}_{t}+\beta_{5} \mathrm{NOIVol}_{t}+\beta_{6} \mathrm{NIVol}_{t}$

$+\beta_{7}$ Size $_{t}+\beta_{8}$ Age $_{t}+\beta_{9}$ Growth $_{t}+\beta_{10}$ Leverage $_{t}+\beta_{11}$ DivPay $_{t}+\beta_{12}$ Profitability $+\beta_{13}$ Rm $_{t}$

$+\varepsilon_{\mathrm{t}}$

\subsection{The REIT Return Regression Model}

\subsubsection{REIT Return}

The REIT return is defined as the REIT total stock return as follows:

REIT Return $t=\frac{\left(\mathrm{P}_{\mathrm{t}}-\mathrm{P}_{\mathrm{t}-1}\right)+\text { Dividend }_{\mathrm{t}}}{\mathrm{P}_{\mathrm{t}-1}}$

where

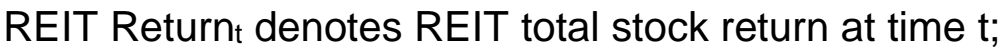

$\mathrm{P}_{\mathrm{t}}$ denotes REIT stock price at time $\mathrm{t}$;

$\mathrm{P}_{\mathrm{t}-1}$ denotes REIT stock price at time $\mathrm{t}-1$;

Dividend ${ }_{t}$ denotes dividend at time $t$. follows:

The general regression model of REIT agency costs on REIT return is as

REIT Return $t=\beta_{0}+\beta_{1}$ FCF $_{t}+\beta_{2}$ Ass T $_{t}+\beta_{3}$ OpeR $_{t}+\beta_{4}$ SGAR $_{t}+\beta_{5}$ NOIVol1 $_{t}+\beta_{6}$ NIVol1 $_{t}$

$+\beta_{7}$ Size $_{t}+\beta_{8}$ Age $_{t}+\beta_{9}$ Growth $_{t}+\beta_{10}$ Leverage $_{t}+\beta_{11}$ DivPay $_{t}+\beta_{12}$ Profitability $+\beta_{13}$ Rm $_{t}$

$+\varepsilon \mathrm{t}$ 


\subsection{Control Variables}

\subsubsection{Size}

Prior studies such as Ooi and Liow (2004) and Lecomte and Ooi (2013) also incorporate firm size that may influence the performance and stock return in REITs. Moreover, Chen et al. (2012) and Hardin III et al. (2009) also contended that the cash holding is negatively associated with size of REITs.

In addition, Ang et al. (2000), Singh et al. (2003), Iskandar et al. (2012), Henry (2010), Florackis et al. (2009), Jelinek et al. (2009) and Hijazi et al. (2011) also use firm size as a control variable in their studies. Thus, the natural log of total assets as the proxy for firm size is included in the regression model. The size is defined as follows:

Size $=\ln ($ Total Assetst $)$

\subsubsection{Age}

Following the Coad, Segarra and Teruel (2013), Jelinek et al. (2009) and Hijazi et al. (2011) and Fleming et al. (2005) also use age as a control variable in their studies. Therefore, firm age is used as a control variable. The age is defined as follows:

Age $_{t}=$ Age at time t.

\subsubsection{Growth}

From the literatures on REITs, it is found that Noguera (2012) uses market-tobook ratio of equity and Lecomte et al. (2013) use market-to-book value in year $t$ as a control variable. Others such as Chen et al. (2012), Hardin III et al. (2009), Hutchinson and Gul (2004) and Chen and Chen (2012) also use growth as a control variable although Baker (1993) and Gul (1999) posit a basic negative association between an exogenous variable, growth opportunities and firm performance.

Thus, the proxy for growth is defined as follows:

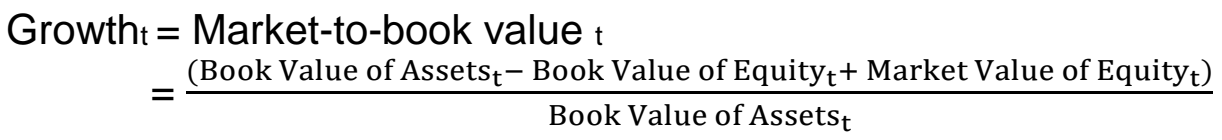

\subsubsection{Leverage}


The literature on REITs such as Chen et al. (2012), Hardin III et al. (2009), Noguera (2012) and Lecomte et al. (2013) also include leverage as a control variable in their regression models.

Other literatures include Myers (1977), Grossman and Hart (1982), Easterbrook (1984), Smith and Watts (1992), Williams (1987), Ang et al. (2000), Singh et al. (2003), Florackis et al. (2009), Fleming et al. (2005), Jelinek et al. (2009), Henry (2010), Hijazi et al. (2011), Iskandar et al. (2012), and Al-Najjar (2013) also contended that financial leverage could influence firm performance. Therefore, leverage is included as a control variable. The leverage is defined as follows:

Leverage $_{t}=$ Debt $_{t} /$ Asset $_{t}$

\subsubsection{Dividend Payout Ratio}

Following the literatures in Cleary (2006), Rozeff (1982), La Porta, Lopez-DeSilanes, Shleifer and Vishny (2000), Bartram, Brown, How and Verhoevan (2008), Easterbrook (1984), Jensen (1986), Zwiebel (1996), Florackis et al. (2009), Feng et al. (2007), John and Knyazeka (2006), John, Knyazeva and Knyazeva (2011), Lee, Gupta, Chen and Lee (2011), DeAngelo, DeAngelo and Stulz (2006), Denis and Osobov (2008), Adjaoud and Ben-Amar (2010) and Henry (2010), the dividend payout ratio is included as a control variable in this study.

Moreover, the REIT regulations require REITs to distribute at least $90 \%$ of total income as dividend for tax exemption. Besides that, REITs are exempted from capital gains tax. Therefore, dividend payout is included as a control variable. The dividend payout ratio is defined as follows:

Dividend Payout Ratio $t=\frac{\text { Total Dividend }_{t}}{\text { Net Income }_{t}}$

\subsubsection{Profitability}

Al-Najjar (2013), Al-Najjar and Belghitar (2011) and Jirapon, Kim and Kim (2011) depict that profitability which has effects on cash holding. Hence, profitability is also included in the regression model as a control variable. The profitability is defined as follows:

Profitability $_{t}=\frac{\text { Net Income After Interest and Taxes }}{t}$

\subsubsection{Market Return}


Market return refers to the return of major stock exchange indices of the respective countries i.e. Tokyo Price Index (TOPIX) for Japan; Straits Times Index (STI) for Singapore; Bursa Malaysia Kuala Lumpur Composite Index (KLCI) for Malaysia and Hang Seng Index (HSI) for Hong Kong. The market return is defined as follows:

$R m_{t}=\frac{X_{t}-X_{t-1}}{X_{t-1}}$

where

$R m_{t}$ denotes market return at time $t$, a control variable;

$\mathrm{X}_{\mathrm{t}}$ denotes market index at time $\mathrm{t}$;

$\mathrm{X}_{\mathrm{t}-1}$ denotes market index at time $\mathrm{t}-1$.

\subsection{RESULTS}

4.1 The Analysis of Free Cash Flow on Asset Turnover Ratio for the REITs in Asia

The results in Table 4.1 show that Model 1, Model 2, Model 3, Model 4, Model 5 and Model 6 passed all the Sargan test and $A R(1)$ and $A R(2)$ tests respectively. 
The free cash flow 1 (FCF1) is significantly positive at $1 \%$ level to asset turnover ratio in Model 1. This is also observed in free cash flow 3 (FCF3) whereby free cash flow 3 (FCF3) is also significantly positive at $1 \%$ level to asset turnover ratio in Model 5 . This is also observed in the robust standard error model in Model 6.

This indicates that free cash flow supports the free cash flow theory. The free cash flow helps to induce agency costs in REITs. Therefore, it is in the same direction of asset turnover ratio.

The results in Table 4.1 also show that free cash flow 2 (FCF2) is significantly negative at $1 \%$ level to asset turnover ratio in Model 3 . This is also observed in the robust standard error model in Model 4. This indicates that free cash flow does not support the free cash flow theory. The free cash flow helps to mitigate agency cost, asset turnover in REITs. Therefore, it is in the opposite direction of asset turnover ratio.

In addition, the lagged asset turnover ratio is also significantly positive at $1 \%$ level to asset turnover ratio in Model 1, Model 3 and Model 5 respectively. This is also observed in the lagged asset turnover ratio in the robust standard error model in Model 2. This indicates that lagged asset turnover ratio also helps to induce agency cost in REITs and it is dynamic in the model.

Besides that, the size and growth are significantly negative to asset turnover respectively in all models except growth in Model 4. This indicates that size and growth help to mitigate and reduce agency costs in REITs.

However, the age is significantly positive to asset turnover in all models. This helps to induce higher agency costs in REITs.

Nonetheless, the profitability shows different signs in the results shown in Table 4.1. The profitability is significantly positive at $1 \%$ level to asset turnover in Model 3 and Model 4 respectively, but it is significantly negative at $1 \%$ level to asset turnover in Model 5.

The leverage and dividend payout ratio are significantly negative at $5 \%$ and $10 \%$ level to asset turnover in Model 3 and Model 1 respectively.

Interestingly, the constant is significantly positive at $1 \%$ level in all models respectively. Thus, this supports the fact that the individual REIT in Asia possesses its own unique characteristics.

Table 4.1 The Results of Two-Step Different GMM and Two-Step Different GMM with Robust Standard Error of Free Cash Flow (FCF) on Asset Turnover Ratio for the REITs in Asia. 


\begin{tabular}{|c|c|c|c|c|c|c|}
\hline Items & $\begin{array}{c}\text { Two-Step } \\
\text { Diff GMM } \\
\\
\text { Model } 1 \\
\text { Asset Turnover } \\
\text { Ratio }\end{array}$ & $\begin{array}{c}\text { Two-Step } \\
\text { Diff GMM with } \\
\text { Robust SE } \\
\text { Model } 2 \\
\text { Asset Turnover } \\
\text { Ratio }\end{array}$ & $\begin{array}{c}\text { Two-Step } \\
\text { Diff GMM } \\
\text { Model } 3 \\
\text { Asset Turnover } \\
\text { Ratio }\end{array}$ & $\begin{array}{c}\text { Two-Step } \\
\text { Diff GMM with } \\
\text { Robust SE } \\
\text { Model } 4 \\
\text { Asset Turnover } \\
\text { Ratio }\end{array}$ & $\begin{array}{c}\text { Two-Step } \\
\text { Diff GMM } \\
\text { Model } 5 \\
\text { Asset Turnover } \\
\text { Ratio }\end{array}$ & $\begin{array}{c}\text { Two-Step } \\
\text { Diff GMM with } \\
\text { Robust SE } \\
\text { Model } 6 \\
\text { Asset Turnover } \\
\text { Ratio }\end{array}$ \\
\hline Lagged 1 & $\begin{array}{l}0.1424379 \\
(4.56)^{* * *}\end{array}$ & $\begin{array}{c}0.1424379 \\
(1.90)^{*}\end{array}$ & $\begin{array}{l}0.1100471 \\
(2.76)^{* * *}\end{array}$ & $\begin{array}{c}0.1100471 \\
(1.40)\end{array}$ & $\begin{array}{l}0.091683 \\
(3.31)^{* * *}\end{array}$ & $\begin{array}{c}0.091683 \\
(1.10)\end{array}$ \\
\hline FCF1 & $\begin{array}{c}0.0726934 \\
(4.32)^{* * *}\end{array}$ & $\begin{array}{c}0.0726934 \\
(1.51) \\
\end{array}$ & & & & \\
\hline FCF2 & & & $\begin{array}{l}-0.0396373 \\
(-5.26)^{* * *}\end{array}$ & $\begin{array}{l}-0.0396373 \\
(-2.65)^{* * *}\end{array}$ & & \\
\hline FCF3 & & & & & $\begin{array}{c}1.28 \mathrm{E}-08 \\
(13.87)^{* * * *}\end{array}$ & $\begin{array}{l}1.28 \mathrm{E}-08 \\
(3.43)^{* * *}\end{array}$ \\
\hline Size & $\begin{array}{l}-0.049586 \\
(-9.75)^{* * *}\end{array}$ & $\begin{array}{l}-0.049586 \\
(-3.25)^{* * *}\end{array}$ & $\begin{array}{l}-0.0378243 \\
(-7.49) * * *\end{array}$ & $\begin{array}{l}-0.0378243 \\
(-2.88)^{* * *}\end{array}$ & $\begin{array}{l}-0.0365156 \\
(-7.56)^{* * *}\end{array}$ & $\begin{array}{c}-0.0365156 \\
(-2.34)^{* *}\end{array}$ \\
\hline Age & $\begin{array}{l}0.0030816 \\
(10.44)^{* * *}\end{array}$ & $\begin{array}{l}0.0030816 \\
(3.54)^{* * *}\end{array}$ & $\begin{array}{l}0.0023402 \\
(6.84)^{* * *}\end{array}$ & $\begin{array}{l}0.0023402 \\
(2.73)^{* * *}\end{array}$ & $\begin{array}{l}0.0021633 \\
(8.40)^{* * *}\end{array}$ & $\begin{array}{c}0.0021633 \\
(2.55)^{* *}\end{array}$ \\
\hline Growth & $\begin{array}{l}-0.0088538 \\
(-5.04)^{* * *}\end{array}$ & $\begin{array}{c}-0.0088538 \\
(-2.20)^{* *}\end{array}$ & $\begin{array}{l}-0.0069441 \\
(-4.10)^{* * *}\end{array}$ & $\begin{array}{c}-0.0069441 \\
(-1.49)\end{array}$ & $\begin{array}{l}-0.0078653 \\
(-6.37)^{* * *}\end{array}$ & $\begin{array}{c}-0.0078653 \\
(-2.40)^{* *}\end{array}$ \\
\hline Leverage & $\begin{array}{c}-0.0001248 \\
(-0.02)\end{array}$ & $\begin{array}{c}-0.0001248 \\
(-0.01)\end{array}$ & $\begin{array}{c}-0.0134247 \\
(-2.39) * *\end{array}$ & $\begin{array}{c}-0.0134247 \\
(-1.31)\end{array}$ & $\begin{array}{c}0.0043644 \\
(0.82)\end{array}$ & $\begin{array}{c}0.0043644 \\
(0.37)\end{array}$ \\
\hline Divpay ratio & $\begin{array}{c}-0.0010485 \\
(-1.94)^{*}\end{array}$ & $\begin{array}{c}-0.0010485 \\
(-0.97)\end{array}$ & $\begin{array}{c}-0.0007171 \\
(-1.20)\end{array}$ & $\begin{array}{c}-0.0007171 \\
(-0.60)\end{array}$ & $\begin{array}{c}-0.0006437 \\
(-1.07)\end{array}$ & $\begin{array}{c}-0.0006437 \\
(-0.47)\end{array}$ \\
\hline Profitability & $\begin{array}{c}-0.0042425 \\
(-1.10)\end{array}$ & $\begin{array}{c}-0.0042425 \\
(-0.40)\end{array}$ & $\begin{array}{l}0.0175047 \\
(15.61)^{* * *}\end{array}$ & $\begin{array}{l}0.0175047 \\
(4.36)^{* * *}\end{array}$ & $\begin{array}{l}-0.0115793 \\
(-3.30)^{* * *}\end{array}$ & $\begin{array}{c}-0.0115793 \\
(-1.45)\end{array}$ \\
\hline Constant & $\begin{array}{l}0.3542277 \\
(12.43)^{* * *}\end{array}$ & $\begin{array}{l}0.3542277 \\
(4.05)^{* * *}\end{array}$ & $\begin{array}{l}0.2950035 \\
(9.97)^{* * *}\end{array}$ & $\begin{array}{l}0.2950035 \\
(3.68)^{* * *}\end{array}$ & $\begin{array}{l}0.2804732 \\
(10.07)^{* * *}\end{array}$ & $\begin{array}{l}0.2804732 \\
(3.09)^{* * *}\end{array}$ \\
\hline Sargan Test & $\begin{array}{l}40.56485 \\
{[0.6197]}\end{array}$ & & $\begin{array}{l}37.61077 \\
{[0.7406]}\end{array}$ & & $\begin{array}{l}42.02215 \\
{[0.5567]}\end{array}$ & \\
\hline $\operatorname{AR}(1)$ & $\begin{array}{c}-3.0158 \\
{[0.0026]^{* *}}\end{array}$ & $\begin{array}{c}-2.7153 \\
{[0.0066]^{* * *}}\end{array}$ & $\begin{array}{c}-2.4518 \\
{[0.0142]^{* *}}\end{array}$ & $\begin{array}{c}-2.2382 \\
{[0.0252]^{* *}}\end{array}$ & $\begin{array}{c}-2.7064 \\
{[0.0068]^{* * *}}\end{array}$ & $\begin{array}{c}-2.4016 \\
{[0.0163]^{* *}}\end{array}$ \\
\hline $\operatorname{AR}(2)$ & $\begin{array}{c}0.86861 \\
{[0.3851]} \\
\end{array}$ & $\begin{array}{l}0.84069 \\
{[0.4005]} \\
\end{array}$ & $\begin{array}{l}0.20755 \\
{[0.8356]} \\
\end{array}$ & $\begin{array}{l}0.19372 \\
{[0.8464]} \\
\end{array}$ & $\begin{array}{l}0.31125 \\
{[0.7556]} \\
\end{array}$ & $\begin{array}{r}0.29346 \\
{[0.7692]} \\
\end{array}$ \\
\hline $\begin{array}{l}\text { No of } \\
\text { Observations } \\
\end{array}$ & 327 & 327 & 270 & 270 & 325 & 325 \\
\hline $\begin{array}{l}\text { No of } \\
\text { Instruments }\end{array}$ & 53 & 53 & 53 & 53 & 53 & 53 \\
\hline
\end{tabular}

Notes: The z-statistics are in parentheses and p-values are in squared brackets. The ${ }^{* * *},{ }^{* *}$, and ${ }^{*}$ indicate significance at $1 \%, 5 \%$ and $10 \%$ level respectively.

\subsection{The Analysis of Free Cash Flow on Operating Expense Ratio for the REITs in Asia}


The results in Table 4.2 show that Model 1, Model 2, Model 3, Model 4, Model 5 and Model 6 passed all the Sargan test and $A R(1)$ and $A R(2)$ tests respectively.

The free cash flow 1 (FCF1) is significantly negative at $1 \%$ level to operating expense ratio in Model 1. This indicates that free cash flow does not support the free cash flow theory. The free cash flow helps to mitigate agency cost, operating expense ratio in REITs. Therefore, it is in the opposite direction of operating expense ratio.

However, the results in Table 4.2 also show that free cash flow 2 (FCF2) is significantly positive at $1 \%$ level to operating expense ratio in Model 3 . This also indicates that free cash flow supports the free cash flow theory. The free cash flow helps to induce agency cost, operating expense ratio in REITs. Therefore, it is in the same direction of operating expense ratio.

Besides that, age, dividend payout ratio and profitability are significantly negative to operating expense ratio respectively in Model 1, Model 3 and Model 5 respectively. This indicates that age, dividend payout ratio and profitability help to mitigate agency cost in REITs.

The growth also shows significantly negative at $5 \%$ level to operating expense ratio in Model 5. The growth reduces operating expense ratio in REITs.

The size is significantly positive to operating expense ratio in Model 1, Model 3 and Model 5 respectively. This is also observed in the robust standard error model in Model 6. The bigger the REITs, the bigger the operating expense ratio in REITs in Asia.

Besides that, the leverage is significantly positive to operating expense ratio in all models respectively. This helps to induce higher agency costs in REITs. Hence, the leverage is one of the major operating expenses in REITs in Asia.

Table 4.2 The Results of Two-Step Different GMM and Two-Step Different GMM with Robust Standard Error of Free Cash Flow (FCF) on Operating Expense Ratio for the REITs in Asia. 


\begin{tabular}{|c|c|c|c|c|c|c|}
\hline Items & $\begin{array}{c}\text { Two-Step } \\
\text { Diff GMM } \\
\text { Model } 1 \\
\text { Operating } \\
\text { Expense Ratio }\end{array}$ & $\begin{array}{c}\text { Two-Step } \\
\text { Diff GMM with } \\
\text { Robust SE } \\
\text { Model } 2 \\
\text { Operating } \\
\text { Expense Ratio }\end{array}$ & $\begin{array}{c}\text { Two-Step } \\
\text { Diff GMM } \\
\text { Model } 3 \\
\text { Operating } \\
\text { Expense Ratio }\end{array}$ & $\begin{array}{c}\text { Two-Step } \\
\text { Diff GMM with } \\
\text { Robust SE } \\
\text { Model } 4 \\
\text { Operating } \\
\text { Expense Ratio }\end{array}$ & $\begin{array}{c}\text { Two-Step } \\
\text { Diff GMM } \\
\text { Model } 5 \\
\text { Operating } \\
\text { Expense Ratio }\end{array}$ & $\begin{array}{c}\text { Two-Step } \\
\text { Diff GMM with } \\
\text { Robust SE } \\
\text { Model } 6 \\
\text { Operating } \\
\text { Expense Ratio }\end{array}$ \\
\hline Lagged 1 & $\begin{array}{c}0.0286263 \\
(1.28) \\
\end{array}$ & $\begin{array}{c}0.0286263 \\
(0.25) \\
\end{array}$ & $\begin{array}{c}0.0272475 \\
(1.22) \\
\end{array}$ & $\begin{array}{c}0.0272475 \\
(0.17) \\
\end{array}$ & $\begin{array}{c}0.0095046 \\
(0.33) \\
\end{array}$ & $\begin{array}{c}0.0095046 \\
(0.09) \\
\end{array}$ \\
\hline FCF1 & $\begin{array}{l}-0.6235739 \\
(-4.23)^{* * *}\end{array}$ & $\begin{array}{c}-0.6235739 \\
(-1.08)\end{array}$ & & & & \\
\hline FCF2 & & & $\begin{array}{l}2.099927 \\
(4.57)^{* * *}\end{array}$ & $\begin{array}{c}2.099927 \\
(0.32)\end{array}$ & & \\
\hline FCF3 & & & & & $\begin{array}{c}\text { 7.33E-09 } \\
(1.04)\end{array}$ & $\begin{array}{c}\text { 7.33E-09 } \\
(0.20)\end{array}$ \\
\hline Size & $\begin{array}{c}0.0695958 \\
(2.85)^{* * *}\end{array}$ & $\begin{array}{c}0.0695958 \\
(1.06) \\
\end{array}$ & $\begin{array}{l}0.0813067 \\
(4.28)^{* * *}\end{array}$ & $\begin{array}{c}0.0813067 \\
(0.35) \\
\end{array}$ & $\begin{array}{l}0.1861276 \\
(10.43)^{* * *} \\
\end{array}$ & $\begin{array}{c}0.1861276 \\
(2.07)^{* *}\end{array}$ \\
\hline Age & $\begin{array}{l}-0.0081495 \\
(-5.17)^{* * *}\end{array}$ & $\begin{array}{c}-0.0081495 \\
(-1.57) \\
\end{array}$ & $\begin{array}{l}-0.0078221 \\
(-5.63) * * *\end{array}$ & $\begin{array}{c}-0.0078221 \\
(-0.46) \\
\end{array}$ & $\begin{array}{l}-0.0114562 \\
(-6.72)^{* * *}\end{array}$ & $\begin{array}{c}-0.0114562 \\
(-1.41) \\
\end{array}$ \\
\hline Growth & $\begin{array}{c}-0.0180924 \\
(-1.43)\end{array}$ & $\begin{array}{c}-0.0180924 \\
(-0.56)\end{array}$ & $\begin{array}{c}-0.0084313 \\
(-0.71)\end{array}$ & $\begin{array}{c}-0.0084313 \\
(-0.21)\end{array}$ & $\begin{array}{c}-0.0173057 \\
(-2.31)^{* *}\end{array}$ & $\begin{array}{c}-0.0173057 \\
(-0.57)\end{array}$ \\
\hline Leverage & $\begin{array}{l}0.3965281 \\
(8.71)^{* * *}\end{array}$ & $\begin{array}{l}0.3965281 \\
(2.97)^{* * *} \\
\end{array}$ & $\begin{array}{l}0.6792638 \\
(29.20)^{* * *} \\
\end{array}$ & $\begin{array}{c}0.6792638 \\
(2.26)^{* *}\end{array}$ & $\begin{array}{l}0.378471 \\
(9.00)^{* * *}\end{array}$ & $\begin{array}{l}0.378471 \\
(2.35)^{* *} \\
\end{array}$ \\
\hline Divpay ratio & $\begin{array}{l}-0.0077499 \\
(-4.89)^{* * *}\end{array}$ & $\begin{array}{c}-0.0077499 \\
(-1.29)\end{array}$ & $\begin{array}{l}-0.0090506 \\
(-3.31)^{* * *}\end{array}$ & $\begin{array}{c}-0.0090506 \\
(-0.67)\end{array}$ & $\begin{array}{l}-0.0097786 \\
(-4.41)^{* * *}\end{array}$ & $\begin{array}{c}-0.0097786 \\
(-1.47)\end{array}$ \\
\hline Profitability & $\begin{array}{l}-0.0874897 \\
(-2.95)^{* * *}\end{array}$ & $\begin{array}{c}-0.0874897 \\
(-1.14) \\
\end{array}$ & $\begin{array}{l}-1.421515 \\
(-4.54) * * * \\
\end{array}$ & $\begin{array}{c}-1.421515 \\
(-0.32) \\
\end{array}$ & $\begin{array}{l}-0.1043057 \\
(-3.10)^{* * *}\end{array}$ & $\begin{array}{c}-0.1043057 \\
(-1.34)\end{array}$ \\
\hline Constant & $\begin{array}{c}0.0179049 \\
(0.13) \\
\end{array}$ & $\begin{array}{c}0.0179049 \\
(0.05) \\
\end{array}$ & $\begin{array}{c}-0.1531299 \\
(-1.31) \\
\end{array}$ & $\begin{array}{c}-0.1531299 \\
(-0.11) \\
\end{array}$ & $\begin{array}{l}-0.6660077 \\
(-5.88)^{* * *}\end{array}$ & $\begin{array}{c}-0.6660077 \\
(-1.25) \\
\end{array}$ \\
\hline Sargan Test & $\begin{array}{r}32.96788 \\
{[0.8886]} \\
\end{array}$ & & $\begin{array}{c}29.41054 \\
{[0.9552]} \\
\end{array}$ & & $\begin{array}{c}35.18546 \\
{[0.8260]}\end{array}$ & \\
\hline $\operatorname{AR}(1)$ & $\begin{array}{c}-1.9581 \\
{[0.0502]^{*}}\end{array}$ & $\begin{array}{c}-1.6767 \\
{[0.0936]^{*}}\end{array}$ & $\begin{array}{c}-1.7037 \\
{[0.0884]^{*}}\end{array}$ & $\begin{array}{c}-1.2914 \\
{[0.1966]}\end{array}$ & $\begin{array}{c}-2.0633 \\
{[0.0391]^{* *}}\end{array}$ & $\begin{array}{c}-1.8158 \\
{[0.0694]^{*}}\end{array}$ \\
\hline $\operatorname{AR}(2)$ & $\begin{array}{c}-0.364 \\
{[0.7159]}\end{array}$ & $\begin{array}{l}-0.31038 \\
{[0.7563]}\end{array}$ & $\begin{array}{l}0.19247 \\
{[0.8474]}\end{array}$ & $\begin{array}{l}0.06174 \\
{[0.9508]}\end{array}$ & $\begin{array}{l}0.63295 \\
{[0.5268]}\end{array}$ & $\begin{array}{l}0.52295 \\
{[0.6010]}\end{array}$ \\
\hline $\begin{array}{l}\text { No of } \\
\text { Observations }\end{array}$ & 203 & 203 & 160 & 160 & 205 & 205 \\
\hline $\begin{array}{l}\text { No of } \\
\text { Instruments }\end{array}$ & 53 & 53 & 53 & 53 & 53 & 53 \\
\hline
\end{tabular}

Notes: The z-statistics are in parentheses and p-values are in squared brackets. The ${ }^{* * *},{ }^{* *}$, and ${ }^{*}$ indicate significance at $1 \%, 5 \%$ and $10 \%$ level respectively.

\subsection{The Analysis of Free Cash Flow on Selling, General and Administrative Expenses Ratio (SGAR) for the REITs in Asia}


The results in Table 4.3 show that Model 1, Model 2, Model 3, Model 4, Model 5 and Model 6 passed all the Sargan test and $A R(1)$ and $A R(2)$ tests respectively.

The results in Table 4.3 show that free cash flow 1 (FCF1) is significantly negative at $1 \%$ level to selling, general and administrative expenses ratio (SGAR) in Model 1. This indicates that free cash flow does not support the free cash flow theory. The free cash flow helps to mitigate and reduce agency cost, SGA ratio in REITs. Therefore, it is in the opposite direction of SGA ratio.

However, the results in Table 4.3 also show that free cash flow 2 (FCF2) is significantly positive at $1 \%$ level to SGA ratio in Model 3 . This also depicts that free cash flow supports the free cash flow theory. The free cash flow helps to induce agency costs in REITs. Therefore, it is in the same direction of SGA ratio.

The lagged selling, general and administrative expenses ratio (lagged SGAR) is significantly positive to selling, general and administrative expenses ratio (SGAR) in all models respectively.

The lagged selling, general and administrative expenses ratio (lagged SGAR) induces higher agency cost in REITs. It is in the same direction of selling, general and administrative expenses ratio (SGAR) and it is dynamic in the model.

However, growth is significantly negative to SGA ratio in Model 1, Model 3 and Model 5 respectively. The growth is in the opposite direction of selling, general and administrative expenses ratio (SGAR). Thus, this indicates that growth helps to mitigate agency costs in REITs.

In addition, age and profitability are also significantly negative to SGA ratio in Model 1 and Model 3 respectively. The age and profitability help to mitigate and reduce agency cost in REITs.

Nonetheless, dividend payout ratio is significantly positive to SGA ratio in all models respectively except Model 2 . This is also observed in size and leverage which are significantly positive to SGA ratio in Model 1 and Model 3 respectively.

Interestingly, dividend payout ratio induces agency cost that is significantly shown in SGA ratio in the results in Table 4.3. This contradicts to the results in Table 4.1 and Table 4.2 whereby dividend payout ratio helps to reduce agency cost in REITs.

Table 4.3 The Results of Two-Step Different GMM and Two-Step Different GMM with Robust Standard Error of Free Cash Flow (FCF) on Selling, General and Administrative Expenses Ratio (SGAR) for the REITs in Asia. 


\begin{tabular}{|c|c|c|c|c|c|c|}
\hline Items & $\begin{array}{c}\text { Two-Step } \\
\text { Diff GMM } \\
\text { Model } 1 \\
\text { SGA Ratio } \\
\end{array}$ & \begin{tabular}{|c|} 
Two-Step \\
Diff GMM with \\
Robust SE \\
Model 2 \\
SGA Ratio \\
\end{tabular} & $\begin{array}{c}\text { Two-Step } \\
\text { Diff GMM } \\
\text { Model } 3 \\
\text { SGA Ratio } \\
\end{array}$ & \begin{tabular}{|c|} 
Two-Step \\
Diff GMM with \\
Robust SE \\
Model 4 \\
SGA Ratio \\
\end{tabular} & $\begin{array}{c}\text { Two-Step } \\
\text { Diff GMM } \\
\text { Model } 5 \\
\text { SGA Ratio } \\
\end{array}$ & \begin{tabular}{|c} 
Two-Step \\
Diff GMM with \\
Robust SE \\
Model 6 \\
SGA Ratio \\
\end{tabular} \\
\hline Lagged 1 & $\begin{array}{l}0.3098802 \\
(10.46)^{* * *}\end{array}$ & $\begin{array}{c}0.3098802 \\
(2.52)^{* *}\end{array}$ & $\begin{array}{l}0.314982 \\
(7.77) * * *\end{array}$ & $\begin{array}{l}0.314982 \\
(2.67) * * *\end{array}$ & $\begin{array}{c}0.2864883 \\
(6.67) * * *\end{array}$ & $\begin{array}{c}0.2864883 \\
(2.23)^{* *}\end{array}$ \\
\hline FCF1 & $\begin{array}{l}-0.3809629 \\
(-2.98) * * *\end{array}$ & $\begin{array}{c}-0.3809629 \\
(-1.38)\end{array}$ & & & & \\
\hline FCF2 & & & $\begin{array}{l}0.5458043 \\
(2.98) * * * \\
\end{array}$ & $\begin{array}{c}0.5458043 \\
(1.07)\end{array}$ & & \\
\hline FCF3 & & & & & $\begin{array}{c}-3.57 \mathrm{E}-09 \\
(-0.16)\end{array}$ & $\begin{array}{c}-3.57 \mathrm{E}-09 \\
(-0.05)\end{array}$ \\
\hline Size & $\begin{array}{c}0.2474204 \\
(3.16)^{* * *}\end{array}$ & $\begin{array}{c}0.2474204 \\
(0.91) \\
\end{array}$ & $\begin{array}{c}-0.0696776 \\
(-0.94) \\
\end{array}$ & $\begin{array}{c}-0.0696776 \\
(-0.39) \\
\end{array}$ & $\begin{array}{c}-0.0087473 \\
(-0.09) \\
\end{array}$ & $\begin{array}{c}-0.0087473 \\
(-0.05) \\
\end{array}$ \\
\hline Age & $\begin{array}{l}-0.0136026 \\
(-3.18) * * *\end{array}$ & $\begin{array}{c}-0.0136026 \\
(-1.08) \\
\end{array}$ & $\begin{array}{c}0.0043043 \\
(1.17) \\
\end{array}$ & $\begin{array}{c}0.0043043 \\
(0.43) \\
\end{array}$ & $\begin{array}{c}0.0023351 \\
(0.46) \\
\end{array}$ & $\begin{array}{c}0.0023351 \\
(0.24) \\
\end{array}$ \\
\hline Growth & $\begin{array}{c}-0.0493006 \\
(-1.67)^{*}\end{array}$ & $\begin{array}{c}-0.0493006 \\
(-0.85) \\
\end{array}$ & $\begin{array}{l}-0.1173097 \\
(-4.97)^{* * *}\end{array}$ & $\begin{array}{c}-0.1173097 \\
(-1.46) \\
\end{array}$ & $\begin{array}{c}-0.0777874 \\
(-2.48)^{* *}\end{array}$ & $\begin{array}{c}-0.0777874 \\
(-1.56) \\
\end{array}$ \\
\hline Leverage & $\begin{array}{c}-0.0334971 \\
(-0.54) \\
\end{array}$ & $\begin{array}{c}-0.0334971 \\
(-0.23)\end{array}$ & $\begin{array}{c}0.2260554 \\
(3.99) * * *\end{array}$ & $\begin{array}{c}0.2260554 \\
(1.50)\end{array}$ & $\begin{array}{c}-0.0061598 \\
(-0.10) \\
\end{array}$ & $\begin{array}{c}-0.0061598 \\
(-0.04)\end{array}$ \\
\hline Divpay ratio & $\begin{array}{c}0.0117048 \\
(1.79)^{*}\end{array}$ & $\begin{array}{c}0.0117048 \\
(0.90)\end{array}$ & $\begin{array}{l}0.0313114 \\
(5.43)^{* * *}\end{array}$ & $\begin{array}{c}0.0313114 \\
(2.06)^{* *}\end{array}$ & $\begin{array}{l}0.0254284 \\
(3.17) * * *\end{array}$ & $\begin{array}{c}0.0254284 \\
(2.36) * *\end{array}$ \\
\hline Profitability & $\begin{array}{c}-0.0028301 \\
(-0.09)\end{array}$ & $\begin{array}{c}-0.0028301 \\
(-0.04)\end{array}$ & $\begin{array}{l}-0.3640482 \\
(-2.80)^{* * *}\end{array}$ & $\begin{array}{c}-0.3640482 \\
(-1.13)\end{array}$ & $\begin{array}{c}-0.027122 \\
(-0.75)\end{array}$ & $\begin{array}{c}-0.027122 \\
(-0.52)\end{array}$ \\
\hline Constant & $\begin{array}{l}-1.278772 \\
(-2.82) * * *\end{array}$ & $\begin{array}{c}-1.278772 \\
(-0.82)\end{array}$ & $\begin{array}{c}0.5019925 \\
(1.14)\end{array}$ & $\begin{array}{c}0.5019925 \\
(0.46)\end{array}$ & $\begin{array}{c}0.1809667 \\
(0.31)\end{array}$ & $\begin{array}{c}0.1809667 \\
(0.18)\end{array}$ \\
\hline Sargan Test & $\begin{array}{l}18.49592 \\
{[0.7301]}\end{array}$ & & $\begin{array}{c}14.59914 \\
{[0.9084]}\end{array}$ & & $\begin{array}{c}13.78204 \\
{[0.8414]}\end{array}$ & \\
\hline $\mathrm{AR}(1)$ & $\begin{array}{c}-1.9434 \\
{[0.0520]^{*}}\end{array}$ & $\begin{array}{l}-1.5543 \\
{[0.1201]}\end{array}$ & $\begin{array}{c}-1.7478 \\
{[0.0805]^{*}}\end{array}$ & $\begin{array}{l}-1.3749 \\
{[0.1692]}\end{array}$ & $\begin{array}{c}-1.7718 \\
{[0.0764]^{*}}\end{array}$ & $\begin{array}{l}-1.3242 \\
{[0.1854]}\end{array}$ \\
\hline $\operatorname{AR}(2)$ & $\begin{array}{c}0.56883 \\
{[0.5695]}\end{array}$ & $\begin{array}{c}0.49991 \\
{[0.6171]}\end{array}$ & $\begin{array}{l}0.37985 \\
{[0.7041]}\end{array}$ & $\begin{array}{l}0.33427 \\
{[0.7382]}\end{array}$ & $\begin{array}{c}0.16646 \\
{[0.8678]}\end{array}$ & $\begin{array}{c}0.14716 \\
{[0.8830]}\end{array}$ \\
\hline $\begin{array}{l}\text { No of } \\
\text { Observations }\end{array}$ & 141 & 141 & 101 & 101 & 139 & 139 \\
\hline $\begin{array}{l}\text { No of } \\
\text { Instruments }\end{array}$ & 32 & 32 & 32 & 32 & 29 & 29 \\
\hline
\end{tabular}

Notes: The z-statistics are in parentheses and p-values are in squared brackets. The ${ }^{* * *},{ }^{* *}$, and ${ }^{*}$ indicate significance at $1 \%, 5 \%$ and $10 \%$ level respectively.

\subsection{The Analysis of Free Cash Flow on Net Operating Income Volatility 1 (NOIVOL1) for the REITs in Asia}


The results in Table 4.4 show that only Model 3, Model 4, Model 5 and Model 6 passed all the Sargan test and $A R(1)$ and $A R(2)$ tests respectively. The Model 1 and Model 2 failed to pass the $A R(2)$ test respectively.

The free cash flow 2 (FCF2) is significantly positive at $1 \%$ level to net operating income volatility 1 (NOIVOL1) in Model 3. This indicates that free cash flow supports the free cash flow theory. The free cash flow helps to induce agency costs in REITs. Therefore, it is in the same direction of NOIVOL1.

However, the results in Table 4.4 also show that free cash flow 3 (FCF3) is significantly negative at $1 \%$ level to NOIVOL1 in Model 5 . This is also observed in the robust standard error model in Model 6. This also provides empirical evidence that free cash flow does not support the free cash flow theory. The free cash flow helps to mitigate and reduce agency costs in REITs. Therefore, it is in the opposite direction of NOIVOL1.

However, the lagged net operating income volatility 1 (lagged NOIVOL1) is significantly positive at $1 \%$ level to net operating income volatility 1 (NOIVOL1) in Model 5.

The lagged net operating income volatility 1 (lagged NOIVOL1) induces higher agency cost in REITs. It is in the same direction of NOIVOL1 and it is dynamic in the model.

Besides that, size is significantly negative to net operating income volatility 1 (NOIVOL1) in Model 3, Model 4, Model 5 and Model 6 respectively. This indicates that size helps to mitigate agency costs in REITs.

The growth is significantly negative to net operating income volatility 1 (NOIVOL1) in Model 3 and Model 4 respectively. However, growth is also significantly positive to net operating income volatility 1 (NOIVOL1) in Model 5 . This indicates that growth can help to mitigate agency cost or induce agency cost in net operating income volatility 1 (NOIVOL1).

The age is significantly positive to NOIVOL1 in Model 3 and Model 4 respectively. The leverage and dividend payout ratio are significantly positive to NOIVOL1 in Model 3 and Model 5 respectively.

Besides that, the profitability is significantly positive to NOIVOL1 in Model 3 and Model 5 respectively. This helps to induce higher agency costs in REITs.

Interestingly, the constant is significantly positive at $1 \%$ level in all the Model 3 , Model 4, Model 5 and Model 6 respectively. Thus, this supports the fact that the individual REIT in Asia possesses its own unique characteristics.

Table 4.4 The Results of Two-Step Different GMM and Two-Step Different GMM with Robust Standard Error of Free Cash Flow (FCF) on Net Operating Income Volatility 1 (NOIVOL1) for the REITs in Asia. 


\begin{tabular}{|c|c|c|c|c|c|c|}
\hline Items & $\begin{array}{c}\text { Two-Step } \\
\text { Diff GMM } \\
\text { Model } 1 \\
\text { NOIVOL1 }\end{array}$ & $\begin{array}{c}\text { Two-Step } \\
\text { Diff GMM with } \\
\text { Robust SE } \\
\text { Model } 2 \\
\text { NOIVOL1 }\end{array}$ & $\begin{array}{c}\text { Two-Step } \\
\text { Diff GMM } \\
\text { Model } 3 \\
\text { NOIVOL1 }\end{array}$ & $\begin{array}{c}\text { Two-Step } \\
\text { Diff GMM with } \\
\text { Robust SE } \\
\text { Model } 4 \\
\text { NOIVOL1 }\end{array}$ & $\begin{array}{c}\text { Two-Step } \\
\text { Diff GMM } \\
\text { Model } 5 \\
\text { NOIVOL1 }\end{array}$ & $\begin{array}{c}\text { Two-Step } \\
\text { Diff GMM with } \\
\text { Robust SE } \\
\text { Model } 6 \\
\text { NOIVOL1 }\end{array}$ \\
\hline Lagged 1 & $\begin{array}{l}0.013552 \\
(5.74)^{* * *}\end{array}$ & $\begin{array}{c}0.013552 \\
(0.65) \\
\end{array}$ & $\begin{array}{c}-0.0068488 \\
(-1.08) \\
\end{array}$ & $\begin{array}{c}-0.0068488 \\
(-0.10)\end{array}$ & $\begin{array}{l}0.0442396 \\
(10.93)^{* * *}\end{array}$ & $\begin{array}{c}0.0442396 \\
(1.52) \\
\end{array}$ \\
\hline FCF1 & $\begin{array}{l}0.4176339 \\
(3.61)^{* * *}\end{array}$ & $\begin{array}{c}0.4176339 \\
(1.07)\end{array}$ & & & & \\
\hline FCF2 & & & $\begin{array}{c}0.35018 \\
(5.54)^{* * *}\end{array}$ & $\begin{array}{c}0.35018 \\
(1.16)\end{array}$ & & \\
\hline FCF3 & & & & & $\begin{array}{c}-8.50 \mathrm{E}-08 \\
(-12.45)^{* * *}\end{array}$ & $\begin{array}{l}-8.50 \mathrm{E}-08 \\
(-2.68)^{* * *}\end{array}$ \\
\hline Size & $\begin{array}{c}-1.71971 \\
(-28.32)^{* * *}\end{array}$ & $\begin{array}{c}-1.71971 \\
(-2.86)^{* * *}\end{array}$ & $\begin{array}{c}-1.928193 \\
(-38.53)^{* * *}\end{array}$ & $\begin{array}{l}-1.928193 \\
(-3.22)^{* * *}\end{array}$ & $\begin{array}{l}-0.5223864 \\
(-25.75)^{* * *}\end{array}$ & $\begin{array}{l}-0.5223864 \\
(-5.10)^{* * *}\end{array}$ \\
\hline Age & $\begin{array}{l}0.0822385 \\
(19.84)^{* * *}\end{array}$ & $\begin{array}{c}0.0822385 \\
(2.13)^{* *}\end{array}$ & $\begin{array}{l}0.0931623 \\
(19.75)^{* * *}\end{array}$ & $\begin{array}{l}0.0931623 \\
(2.68) * * *\end{array}$ & $\begin{array}{c}0.0006777 \\
(0.51) \\
\end{array}$ & $\begin{array}{c}0.0006777 \\
(0.12) \\
\end{array}$ \\
\hline Growth & $\begin{array}{l}-0.1217005 \\
(-8.52)^{* * *}\end{array}$ & $\begin{array}{c}-0.1217005 \\
(-1.38)\end{array}$ & $\begin{array}{l}-0.1316425 \\
(-14.79) * * *\end{array}$ & $\begin{array}{c}-0.1316425 \\
(-1.91)^{*}\end{array}$ & $\begin{array}{c}0.0095885 \\
(1.92)^{*}\end{array}$ & $\begin{array}{c}0.0095885 \\
(0.48)\end{array}$ \\
\hline Leverage & $\begin{array}{l}0.1977403 \\
(3.79) * * *\end{array}$ & $\begin{array}{c}0.1977403 \\
(1.29)\end{array}$ & $\begin{array}{c}0.1949575 \\
(5.91)^{* * *}\end{array}$ & $\begin{array}{c}0.1949575 \\
(1.40)\end{array}$ & $\begin{array}{c}0.0153594 \\
(0.62)\end{array}$ & $\begin{array}{c}0.0153594 \\
(0.21)\end{array}$ \\
\hline Divpay ratio & $\begin{array}{l}0.0160456 \\
(2.67) * * * \\
\end{array}$ & $\begin{array}{c}0.0160456 \\
(0.99) \\
\end{array}$ & $\begin{array}{c}0.002045 \\
(0.40) \\
\end{array}$ & $\begin{array}{c}0.002045 \\
(0.14) \\
\end{array}$ & $\begin{array}{l}0.0083282 \\
(3.72)^{* * *}\end{array}$ & $\begin{array}{c}0.0083282 \\
(1.26) \\
\end{array}$ \\
\hline Profitability & $\begin{array}{c}0.1791466 \\
(6.50)^{* * *}\end{array}$ & $\begin{array}{c}0.1791466 \\
(1.93)^{*}\end{array}$ & $\begin{array}{l}0.0802021 \\
(6.38)^{* * *}\end{array}$ & $\begin{array}{c}0.0802021 \\
(1.63)\end{array}$ & $\begin{array}{l}0.0388206 \\
(2.67)^{* * *}\end{array}$ & $\begin{array}{c}0.0388206 \\
(0.72)\end{array}$ \\
\hline Constant & $\begin{array}{c}10.31888 \\
(26.74)^{* * *}\end{array}$ & $\begin{array}{l}10.31888 \\
(2.85)^{* * *}\end{array}$ & $\begin{array}{c}11.65934 \\
(34.12)^{* * *}\end{array}$ & $\begin{array}{l}11.65934 \\
(3.24)^{* * *}\end{array}$ & $\begin{array}{c}3.332545 \\
(29.83)^{* * *}\end{array}$ & $\begin{array}{l}3.332545 \\
(5.59)^{* * *}\end{array}$ \\
\hline Sargan Test & $\begin{array}{l}42.64221 \\
{[0.5299]} \\
\end{array}$ & & $\begin{array}{c}25.63547 \\
{[0.9878]} \\
\end{array}$ & & $\begin{array}{l}40.29749 \\
{[0.6311]} \\
\end{array}$ & \\
\hline $\operatorname{AR}(1)$ & $\begin{array}{c}-2.5182 \\
{[0.0118]^{* *}}\end{array}$ & $\begin{array}{c}-2.4574 \\
{[0.0140]^{* *}}\end{array}$ & $\begin{array}{c}-2.2914 \\
{[0.0219]^{* *}}\end{array}$ & $\begin{array}{c}-2.2335 \\
{[0.0255]^{* *}}\end{array}$ & $\begin{array}{c}-1.3166 \\
{[0.1880]}\end{array}$ & $\begin{array}{c}-1.3089 \\
{[0.1906]}\end{array}$ \\
\hline $\operatorname{AR}(2)$ & $\begin{array}{c}1.8102 \\
{[0.0703]^{*}}\end{array}$ & $\begin{array}{c}1.6898 \\
{[0.0911]^{*}}\end{array}$ & $\begin{array}{l}0.83717 \\
{[0.4025]}\end{array}$ & $\begin{array}{l}0.79974 \\
{[0.4239]}\end{array}$ & $\begin{array}{l}0.79754 \\
{[0.4251]}\end{array}$ & $\begin{array}{l}0.67753 \\
{[0.4981]}\end{array}$ \\
\hline $\begin{array}{l}\text { No of } \\
\text { Observations } \\
\end{array}$ & 327 & 327 & 267 & 267 & 323 & 323 \\
\hline $\begin{array}{l}\text { No of } \\
\text { Instruments }\end{array}$ & 53 & 53 & 53 & 53 & 53 & 53 \\
\hline
\end{tabular}

Notes: The $z$-statistics are in parentheses and $p$-values are in squared brackets. The ${ }^{* * *},{ }^{* *}$, and ${ }^{*}$ indicate significance at $1 \%, 5 \%$ and $10 \%$ level respectively.

\subsection{The Analysis of Free Cash Flow on Net Income Volatility 1 (NIVOL1) for the REITs in Asia}


The results in Table 4.5 show that only Model 1 and Model 2 passed all the Sargan test and $A R(1)$ and $A R(2)$ tests respectively. The Model 3, Model 4, Model 5 and Model 6 failed to pass the AR(2) test respectively.

The free cash flow 1 (FCF1) is significantly negative at $1 \%$ level to net income volatility 1 (NIVOL1) in Model 1. This indicates that free cash flow does not support the free cash flow theory. The free cash flow helps to mitigate agency costs in REITs. Therefore, it is in the opposite direction of NIVOL1.

However, the lagged net income volatility 1 (lagged NIVOL1) is significantly positive at $1 \%$ level to net income volatility 1 (NIVOL1) in Model 1 . The lagged net income volatility 1 (lagged NIVOL1) helps to induce higher agency cost in REITs. It is in the same direction of NIVOL1 and it is dynamic in the model.

Besides that, size, growth and profitability are significantly negative to net income volatility 1 (NIVOL1) respectively in Model 1 . This indicates that bigger REITs can help to mitigate and reduce agency cost, net income volatility 1 (NIVOL1) in REITs.

The age, leverage and dividend payout ratio are significantly positive to net income volatility 1 (NIVOL1) in Model 1 and Model 2 respectively. The age, leverage and dividend payout ratio help to induce higher agency cost, net income volatility 1 (NIVOL1) in REITs in Asia.

Interestingly, the constant is significantly positive at $1 \%$ level in Model 1 and Model 2 respectively. Thus, this supports the fact that the individual REIT in Asia possesses its own unique characteristics.

Table 4.5 The Results of Two-Step Different GMM and Two-Step Different GMM with Robust Standard Error of Free Cash Flow (FCF) on Net Income Volatility 1 (NIVOL1) for the REITs in Asia. 


\begin{tabular}{|c|c|c|c|c|c|c|}
\hline Items & $\begin{array}{c}\text { Two-Step } \\
\text { Diff GMM } \\
\text { Model } 1 \\
\text { NIVOL1 } \\
\end{array}$ & \begin{tabular}{|c|} 
Two-Step \\
Diff GMM with \\
Robust SE \\
Model 2 \\
NIVOL1 \\
\end{tabular} & $\begin{array}{c}\text { Two-Step } \\
\text { Diff GMM } \\
\text { Model } 3 \\
\text { NIVOL1 } \\
\end{array}$ & \begin{tabular}{|c|} 
Two-Step \\
Diff GMM with \\
Robust SE \\
Model 4 \\
NIVOL1 \\
\end{tabular} & $\begin{array}{c}\text { Two-Step } \\
\text { Diff GMM } \\
\text { Model } 5 \\
\text { NIVOL1 } \\
\end{array}$ & $\begin{array}{c}\text { Two-Step } \\
\text { Diff GMM with } \\
\text { Robust SE } \\
\text { Model } 6 \\
\text { NIVOL1 } \\
\end{array}$ \\
\hline Lagged 1 & $\begin{array}{l}0.1382761 \\
(31.05)^{* * *}\end{array}$ & $\begin{array}{c}0.1382761 \\
(1.57) \\
\end{array}$ & $\begin{array}{l}0.2823109 \\
(97.40)^{* * *}\end{array}$ & $\begin{array}{l}0.2823109 \\
(5.39)^{* * *}\end{array}$ & $\begin{array}{l}0.0882741 \\
(24.17)^{* * *}\end{array}$ & $\begin{array}{c}0.0882741 \\
(1.29)\end{array}$ \\
\hline FCF1 & $\begin{array}{c}-1.86969 \\
(-5.32)^{* * *}\end{array}$ & $\begin{array}{c}-1.86969 \\
(-1.23)\end{array}$ & & & & \\
\hline FCF2 & & & $\begin{array}{l}0.8079871 \\
(19.90)^{* * *}\end{array}$ & $\begin{array}{c}0.8079871 \\
(1.99)^{* *}\end{array}$ & & \\
\hline FCF3 & & & & & $\begin{array}{c}-2.30 \mathrm{E}-07 \\
(-30.26)^{* * *}\end{array}$ & $\begin{array}{l}-2.30 \mathrm{E}-07 \\
(-2.63)^{* * *}\end{array}$ \\
\hline Size & $\begin{array}{c}-2.078866 \\
(-56.78)^{* * *}\end{array}$ & $\begin{array}{l}-2.078866 \\
(-5.79)^{* * *}\end{array}$ & $\begin{array}{c}-1.541096 \\
(-34.27)^{* * *}\end{array}$ & $\begin{array}{l}-1.541096 \\
(-5.64)^{* * *}\end{array}$ & $\begin{array}{c}-1.273249 \\
(-27.91)^{* * *}\end{array}$ & $\begin{array}{l}-1.273249 \\
(-3.16)^{* * *}\end{array}$ \\
\hline Age & $\begin{array}{l}0.0403683 \\
(11.39)^{* * *}\end{array}$ & $\begin{array}{c}0.0403683 \\
(1.76)^{*}\end{array}$ & $\begin{array}{l}0.0584222 \\
(17.99)^{* * * *}\end{array}$ & $\begin{array}{l}0.0584222 \\
(3.40)^{* * *}\end{array}$ & $\begin{array}{c}0.0020803 \\
(0.75)\end{array}$ & $\begin{array}{c}0.0020803 \\
(0.08)\end{array}$ \\
\hline Growth & $\begin{array}{l}-0.1097602 \\
(-9.18)^{* * *}\end{array}$ & $\begin{array}{c}-0.1097602 \\
(-1.02)\end{array}$ & $\begin{array}{l}-0.2632299 \\
(-42.81)^{* * *}\end{array}$ & $\begin{array}{c}-0.2632299 \\
(-2.26)^{* *}\end{array}$ & $\begin{array}{l}-0.1035873 \\
(-14.42)^{* * *}\end{array}$ & $\begin{array}{c}-0.1035873 \\
(-1.04)\end{array}$ \\
\hline Leverage & $\begin{array}{l}0.9321038 \\
(11.18)^{* * *}\end{array}$ & $\begin{array}{c}0.9321038 \\
(1.86)^{*}\end{array}$ & $\begin{array}{l}0.1195812 \\
(5.98)^{* * *}\end{array}$ & $\begin{array}{c}0.1195812 \\
(0.43)\end{array}$ & $\begin{array}{l}0.6047024 \\
(10.91)^{* * *}\end{array}$ & $\begin{array}{c}0.6047024 \\
(1.63)\end{array}$ \\
\hline Divpay ratio & $\begin{array}{l}0.0519854 \\
(7.30)^{* * *}\end{array}$ & $\begin{array}{c}0.0519854 \\
(1.81)^{*}\end{array}$ & $\begin{array}{l}0.0155246 \\
(5.39)^{* * *}\end{array}$ & $\begin{array}{c}0.0155246 \\
(0.51)\end{array}$ & $\begin{array}{l}0.0366149 \\
(18.88)^{* * *}\end{array}$ & $\begin{array}{c}0.0366149 \\
(1.24)\end{array}$ \\
\hline Profitability & $\begin{array}{l}-0.0666648 \\
(-3.38)^{* * *}\end{array}$ & $\begin{array}{c}-0.0666648 \\
(-0.20)\end{array}$ & $\begin{array}{l}-0.5891334 \\
(-85.70)^{* * *}\end{array}$ & $\begin{array}{l}-0.5891334 \\
(-5.18)^{* * *}\end{array}$ & $\begin{array}{l}-0.2146349 \\
(-13.75)^{* * *}\end{array}$ & $\begin{array}{c}-0.2146349 \\
(-0.82)\end{array}$ \\
\hline Constant & $\begin{array}{c}12.59365 \\
(66.43)^{* * *}\end{array}$ & $\begin{array}{l}12.59365 \\
(6.29)^{* * *}\end{array}$ & $\begin{array}{c}9.742419 \\
(34.65)^{* * *}\end{array}$ & $\begin{array}{l}9.742419 \\
(5.96)^{* * *}\end{array}$ & $\begin{array}{c}8.092476 \\
(32.45)^{* * *}\end{array}$ & $\begin{array}{l}8.092476 \\
(3.56)^{* * *}\end{array}$ \\
\hline Sargan Test & $\begin{array}{c}43.55657 \\
{[0.4905]} \\
\end{array}$ & & $\begin{array}{l}43.49605 \\
{[0.4931]} \\
\end{array}$ & & $\begin{array}{l}50.1648 \\
{[0.2422]}\end{array}$ & \\
\hline $\operatorname{AR}(1)$ & $\begin{array}{c}-1.413 \\
{[0.1576]}\end{array}$ & $\begin{array}{l}-1.2988 \\
{[0.1940]}\end{array}$ & $\begin{array}{l}-1.6447 \\
{[0.1000]}\end{array}$ & $\begin{array}{l}-1.6306 \\
{[0.1030]}\end{array}$ & $\begin{array}{l}-1.0846 \\
{[0.2781]}\end{array}$ & $\begin{array}{l}-1.0576 \\
{[0.2902]}\end{array}$ \\
\hline $\operatorname{AR}(2)$ & $\begin{array}{l}-0.11449 \\
{[0.9089]}\end{array}$ & $\begin{array}{l}-0.11352 \\
{[0.9096]}\end{array}$ & $\begin{array}{c}1.6782 \\
{[0.0933]^{*}}\end{array}$ & $\begin{array}{c}1.6559 \\
{[0.0977]^{*}}\end{array}$ & $\begin{array}{c}1.8901 \\
{[0.0587]^{*}}\end{array}$ & $\begin{array}{c}1.8318 \\
{[0.0670]^{*}}\end{array}$ \\
\hline $\begin{array}{l}\text { No of } \\
\text { Observations }\end{array}$ & 327 & 327 & 269 & 269 & 323 & 323 \\
\hline $\begin{array}{l}\text { No of } \\
\text { Instruments }\end{array}$ & 53 & 53 & 53 & 53 & 53 & 53 \\
\hline
\end{tabular}

Notes: The z-statistics are in parentheses and $p$-values are in squared brackets. The ${ }^{* * *},{ }^{* *}$, and ${ }^{*}$ indicate significance at $1 \%, 5 \%$ and $10 \%$ level respectively.

\subsection{The Analysis of Free Cash Flow and Agency Costs on Return on Assets (ROA) for the REITs in Asia}


The results in Table 4.6 show that Model 1, Model 2, Model 3, Model 4, Model 5 and Model 6 passed all the Sargan test and $A R(1)$ and $A R(2)$ tests respectively.

The results in Table 4.6 show that free cash flow 2 (FCF2) is significantly positive to ROA at in Model 3. This is also observed in the robust standard error model in Model 4. This indicates that free cash flow has positive impact on return on assets (ROA) in REITs in Asia. This highlights the complexity of the capital market whereby the REITs' unitholders also perceive cash flow as an important element that would assert positive impacts on the return of assets (ROA) in the capital market.

The asset turnover ratio is significantly negative to ROA in Model 3 and operating expense ratio is also significantly negative to ROA in Model 1 respectively. These are the agency costs in REITs. Hence, these signs are in the opposite direction of ROA.

Interestingly, the net operating income volatility 1 (NOIVOL1) is significantly positive to ROA in Model 5.

Nonetheless, the net income volatility 1 (NIVOL1) shows dual directions in the results in Table 4.6. In Model 1, the net income volatility 1 (NIVOL1) is significantly positive at $10 \%$ level to ROA but the net income volatility 1 (NIVOL1) is significantly negative at $1 \%$ level to ROA in Model 5.

The profitability is significantly positive at $1 \%$ level to ROA in Model 1, Model 2 , Model 5 and Model 6 respectively. The profitability enhances the return on assets (ROA). Thus, it is in the same direction of ROA.

However, growth and dividend payout ratio are significantly negative to ROA in Model 1, Model 2 and Model 5 respectively. The growth is consistent with Baker (1993) and Gul (1999). The dividend payout ratio is the agency costs in REITs. Hence, the dividend payout ratio is in the opposite direction of ROA.

Besides that, the age is significantly negative to ROA in Model 1. This indicates that older REITs decrease in performance, return on assets (ROA). Therefore, it is in the opposite direction of ROA.

Table 4.6 The Results of Two-Step Different GMM and Two-Step Different GMM with Robust Standard Error of Free Cash Flow and Agency Costs on Return on Assets (ROA) for the REITs in Asia. 


\begin{tabular}{|c|c|c|c|c|c|c|}
\hline Items & $\begin{array}{c}\text { Two-Step } \\
\text { Diff GMM } \\
\text { Model } 1 \\
\text { ROA }\end{array}$ & $\begin{array}{c}\text { Two-Step } \\
\text { Diff GMM with } \\
\text { Robust SE } \\
\text { Model } 2 \\
\text { ROA }\end{array}$ & $\begin{array}{c}\text { Two-Step } \\
\text { Diff GMM } \\
\text { Model } 3 \\
\text { ROA }\end{array}$ & \begin{tabular}{|c} 
Two-Step \\
Diff GMM with \\
Robust SE \\
Model 4 \\
ROA
\end{tabular} & $\begin{array}{c}\text { Two-Step } \\
\text { Diff GMM } \\
\text { Model } 5 \\
\text { ROA }\end{array}$ & \begin{tabular}{|c} 
Two-Step \\
Diff GMM with \\
Robust SE \\
Model 6 \\
ROA
\end{tabular} \\
\hline Lagged 1 & $\begin{array}{c}-0.0204552 \\
(-1.29) \\
\end{array}$ & $\begin{array}{c}-0.0204552 \\
(-1.22) \\
\end{array}$ & $\begin{array}{c}0.0046951 \\
(0.33) \\
\end{array}$ & $\begin{array}{c}0.0046951 \\
(0.13) \\
\end{array}$ & $\begin{array}{c}-0.0161308 \\
(-1.51)\end{array}$ & $\begin{array}{c}-0.0161308 \\
(-0.49)\end{array}$ \\
\hline FCF1 & $\begin{array}{c}-1.957561 \\
(-0.11)\end{array}$ & $\begin{array}{c}-1.957561 \\
(-0.09)\end{array}$ & & & & \\
\hline FCF2 & & & $\begin{array}{l}100.7076 \\
(4.29) * * *\end{array}$ & $\begin{array}{l}100.7076 \\
(2.14)^{* *}\end{array}$ & & \\
\hline FCF3 & & & & & $\begin{array}{c}1.53 \mathrm{E}-06 \\
(0.87) \\
\end{array}$ & $\begin{array}{c}1.53 \mathrm{E}-06 \\
(0.52)\end{array}$ \\
\hline $\begin{array}{l}\text { Asset Turnover } \\
\text { Ratio } \\
\end{array}$ & $\begin{array}{c}-18.12584 \\
(-0.43) \\
\end{array}$ & $\begin{array}{c}-18.12584 \\
(-0.26) \\
\end{array}$ & $\begin{array}{l}-88.90574 \\
(-2.69) * * * \\
\end{array}$ & $\begin{array}{c}-88.90574 \\
(-1.38) \\
\end{array}$ & $\begin{array}{c}-19.14057 \\
(-0.53) \\
\end{array}$ & $\begin{array}{c}-19.14057 \\
(-0.29) \\
\end{array}$ \\
\hline \begin{tabular}{|l|} 
Operating \\
Expense Ratio \\
\end{tabular} & $\begin{array}{l}-9.107299 \\
(-2.47)^{* *} \\
\end{array}$ & $\begin{array}{c}-9.107299 \\
(-1.42) \\
\end{array}$ & $\begin{array}{c}-1.308206 \\
(-0.25) \\
\end{array}$ & $\begin{array}{c}-1.308206 \\
(-0.23) \\
\end{array}$ & $\begin{array}{c}-7.419763 \\
(-1.64) \\
\end{array}$ & $\begin{array}{c}-7.419763 \\
(-0.80) \\
\end{array}$ \\
\hline SGA Ratio & $\begin{array}{c}3.019426 \\
(0.45) \\
\end{array}$ & $\begin{array}{c}3.019426 \\
(0.35) \\
\end{array}$ & $\begin{array}{c}-1.147093 \\
(-0.31) \\
\end{array}$ & $\begin{array}{c}-1.147093 \\
(-0.29) \\
\end{array}$ & $\begin{array}{c}2.96677 \\
(0.22) \\
\end{array}$ & $\begin{array}{c}2.96677 \\
(0.12) \\
\end{array}$ \\
\hline NOIVOL1 & $\begin{array}{c}-8.331788 \\
(-1.28) \\
\end{array}$ & $\begin{array}{c}-8.331788 \\
(-0.88) \\
\end{array}$ & $\begin{array}{c}-12.29551 \\
(-0.52) \\
\end{array}$ & $\begin{array}{c}-12.29551 \\
(-0.25) \\
\end{array}$ & $\begin{array}{l}8.335173 \\
(2.25)^{* *} \\
\end{array}$ & $\begin{array}{c}8.335173 \\
(0.87) \\
\end{array}$ \\
\hline NIVOL1 & $\begin{array}{c}2.028084 \\
(1.88)^{*} \\
\end{array}$ & $\begin{array}{c}2.028084 \\
(1.09) \\
\end{array}$ & $\begin{array}{c}0.1699292 \\
(0.08) \\
\end{array}$ & $\begin{array}{c}0.1699292 \\
(0.04) \\
\end{array}$ & $\begin{array}{l}-1.544164 \\
(-3.20)^{* * *} \\
\end{array}$ & $\begin{array}{c}-1.544164 \\
(-1.07) \\
\end{array}$ \\
\hline Size & $\begin{array}{c}7.297152 \\
(1.44) \\
\end{array}$ & $\begin{array}{c}7.297152 \\
(0.87)\end{array}$ & $\begin{array}{c}-6.270608 \\
(-1.02) \\
\end{array}$ & $\begin{array}{c}-6.270608 \\
(-0.51) \\
\end{array}$ & $\begin{array}{c}2.950929 \\
(0.43) \\
\end{array}$ & $\begin{array}{c}2.950929 \\
(0.30) \\
\end{array}$ \\
\hline Age & $\begin{array}{c}-0.4685077 \\
(-2.05)^{* *}\end{array}$ & $\begin{array}{c}-0.4685077 \\
(-1.33)\end{array}$ & $\begin{array}{c}-0.034653 \\
(-0.14)\end{array}$ & $\begin{array}{c}-0.034653 \\
(-0.07)\end{array}$ & $\begin{array}{c}-0.2949372 \\
(-0.76)\end{array}$ & $\begin{array}{c}-0.2949372 \\
(-0.57)\end{array}$ \\
\hline Growth & $\begin{array}{l}-3.120714 \\
(-2.15) * *\end{array}$ & $\begin{array}{c}-3.120714 \\
(-1.77)^{*}\end{array}$ & $\begin{array}{c}-2.096912 \\
(-0.91) \\
\end{array}$ & $\begin{array}{c}-2.096912 \\
(-0.52)\end{array}$ & $\begin{array}{l}-3.768229 \\
(-2.17)^{* *}\end{array}$ & $\begin{array}{c}-3.768229 \\
(-1.20) \\
\end{array}$ \\
\hline Leverage & $\begin{array}{c}-6.476518 \\
(-0.65)\end{array}$ & $\begin{array}{c}-6.476518 \\
(-0.41)\end{array}$ & $\begin{array}{c}0.9761193 \\
(0.23)\end{array}$ & $\begin{array}{c}0.9761193 \\
(0.12)\end{array}$ & $\begin{array}{c}12.3588 \\
(0.87)\end{array}$ & $\begin{array}{c}12.3588 \\
(0.45)\end{array}$ \\
\hline Divpay ratio & $\begin{array}{l}-0.7209237 \\
(-3.93) * * *\end{array}$ & $\begin{array}{c}-0.7209237 \\
(-2.54) * * \\
\end{array}$ & $\begin{array}{c}0.2068655 \\
(0.66) \\
\end{array}$ & $\begin{array}{c}0.2068655 \\
(0.36) \\
\end{array}$ & $\begin{array}{c}-0.6070145 \\
(-2.15)^{* *}\end{array}$ & $\begin{array}{c}-0.6070145 \\
(-1.09) \\
\end{array}$ \\
\hline Profitability & $\begin{array}{c}68.85905 \\
(26.48)^{* * *} \\
\end{array}$ & $\begin{array}{c}68.85905 \\
(17.38)^{* * *} \\
\end{array}$ & $\begin{array}{c}0.931617 \\
(0.06) \\
\end{array}$ & $\begin{array}{c}0.931617 \\
(0.03)\end{array}$ & $\begin{array}{c}73.14286 \\
(19.44) * * * \\
\end{array}$ & $\begin{array}{l}73.14286 \\
(9.51)^{* * * *} \\
\end{array}$ \\
\hline \begin{tabular}{|l|} 
Constant \\
\end{tabular} & $\begin{array}{c}-29.53807 \\
(-0.90) \\
\end{array}$ & $\begin{array}{c}-29.53807 \\
(-0.53) \\
\end{array}$ & $\begin{array}{c}49.10791 \\
(1.15) \\
\end{array}$ & $\begin{array}{c}49.10791 \\
(0.58) \\
\end{array}$ & $\begin{array}{c}-10.35494 \\
(-0.24) \\
\end{array}$ & $\begin{array}{c}-10.35494 \\
(-0.16) \\
\end{array}$ \\
\hline Sargan Test & $\begin{array}{l}4.227363 \\
{[1.0000]} \\
\end{array}$ & & $\begin{array}{l}3.674241 \\
{[1.0000]} \\
\end{array}$ & & $\begin{array}{l}11.15649 \\
{[0.9988]} \\
\end{array}$ & \\
\hline$\overline{A R(1)}$ & $\begin{array}{c}-1.5006 \\
{[0.1335]} \\
\end{array}$ & $\begin{array}{c}-1.4751 \\
{[0.1402]} \\
\end{array}$ & $\begin{array}{c}-2.0486 \\
{[0.0405]^{* *}} \\
\end{array}$ & $\begin{array}{c}-2.0011 \\
{[0.0454]^{* *}} \\
\end{array}$ & $\begin{array}{c}-1.8529 \\
{[0.0639]^{*}}\end{array}$ & $\begin{array}{c}-1.7864 \\
{[0.0740]^{*}} \\
\end{array}$ \\
\hline $\operatorname{AR}(2)$ & $\begin{array}{l}-0.48589 \\
{[0.6270]} \\
\end{array}$ & $\begin{array}{l}-0.42909 \\
{[0.6679]} \\
\end{array}$ & $\begin{array}{c}1.2525 \\
{[0.2104]} \\
\end{array}$ & $\begin{array}{c}1.0398 \\
{[0.2984]} \\
\end{array}$ & $\begin{array}{l}0.81249 \\
{[0.4165]} \\
\end{array}$ & $\begin{array}{l}0.77215 \\
{[0.4400]} \\
\end{array}$ \\
\hline \begin{tabular}{|l|} 
No of \\
Observations \\
\end{tabular} & 79 & 79 & 60 & 60 & 84 & 84 \\
\hline $\begin{array}{l}\text { No of } \\
\text { Instruments }\end{array}$ & 45 & 45 & 41 & 41 & 43 & 43 \\
\hline
\end{tabular}

Notes: The z-statistics are in parentheses and $p$-values are in squared brackets. The ${ }^{* * *},{ }^{* *}$, and ${ }^{*}$ indicate significance at $1 \%, 5 \%$ and $10 \%$ level respectively.

\subsection{The Analysis of Free Cash Flow and Agency Costs on Return on Equity (ROE) for the REITs in Asia}


The results in Table 4.7 show that Model 1, Model 2, Model 3, Model 5 and Model 6 passed all the Sargan test and $A R(1)$ and $A R(2)$ tests respectively.

The results in Table 4.7 show that free cash flow is not significant to return on equity (ROE) at all in all models respectively.

Nevertheless, the lagged return on equity (lagged ROE) is significantly negative to return on equity (ROE) in Model 1, Model 2 and Model 5 respectively. The lagged return on equity (lagged $\mathrm{ROE}$ ) asserts a significantly negative relationship on return on equity (ROE) and it is dynamic in the model.

In addition, asset turnover ratio, operating expense ratio and net operating income volatility 1 (NOIVOL 1 ) are significantly negative to return on equity (ROE) in Model 3 and Model 1 respectively. These are the agency costs in REITs. Hence, there are in opposite direction of return on equity (ROE).

Nonetheless, the net income volatility 1 (NIVOL1) postulates $10 \%$ significance to the return on equity (ROE) in REITs.

The profitability is significantly positive at $1 \%$ level to return on equity (ROE) in all models respectively. The profitability enhances the return on equity (ROE). Thus, it is in the same direction of ROE. This is identical to the results of profitability in Table 4.6

However, the growth is significantly negative to return on equity (ROE) in Model 1, Model 5 and Model 6 respectively. This indicates that growth has negative effect on return on equity (ROE). Therefore, it is in the opposite direction of return on equity (ROE). This is consistent with Baker (1993) and Gul (1999).

In addition, size and leverage are also significantly negative to return on equity (ROE) respectively. The bigger the REITs, the return on equity is lesser. The leverage is the agency cost in REITs. Thus, it is in the opposite direction of return on equity (ROE).

Table 4.7 The Results of Two-Step Different GMM and Two-Step Different GMM with Robust Standard Error of Free Cash Flow and Agency Costs on Return on Equity (ROE) for the REITs in Asia. 


\begin{tabular}{|c|c|c|c|c|c|}
\hline Items & $\begin{array}{c}\text { Two-Step } \\
\text { Diff GMM } \\
\text { Model } 1 \\
\text { ROE }\end{array}$ & $\begin{array}{c}\text { Two-Step } \\
\text { Diff GMM with } \\
\text { Robust SE } \\
\text { Model } 2 \\
\text { ROE }\end{array}$ & $\begin{array}{c}\text { Two-Step } \\
\text { Diff GMM } \\
\text { Model } 3 \\
\text { ROE }\end{array}$ & $\begin{array}{c}\text { Two-Step } \\
\text { Diff GMM } \\
\text { Model } 5 \\
\text { ROE }\end{array}$ & $\begin{array}{c}\text { Two-Step } \\
\text { Diff GMM with } \\
\text { Robust SE } \\
\text { Model } 6 \\
\text { ROE }\end{array}$ \\
\hline Lagged 1 & $\begin{array}{l}-0.0551067 \\
(-2.95)^{* * *} *\end{array}$ & $\begin{array}{c}-0.0551067 \\
(-1.77)^{*} \\
\end{array}$ & $\begin{array}{c}-0.0254284 \\
(-1.31)\end{array}$ & $\begin{array}{l}-0.0530131 \\
(-2.65) * * *\end{array}$ & $\begin{array}{c}-0.0530131 \\
(-1.22) \\
\end{array}$ \\
\hline FCF1 & $\begin{array}{c}-8.909087 \\
(-1.01) \\
\end{array}$ & $\begin{array}{c}-8.909087 \\
(-0.69) \\
\end{array}$ & & & \\
\hline FCF2 & & & $\begin{array}{c}8.30386 \\
(0.36)\end{array}$ & & \\
\hline FCF3 & & & & $\begin{array}{c}1.46 \mathrm{E}-07 \\
(0.18)\end{array}$ & $\begin{array}{c}1.46 \mathrm{E}-07 \\
(0.10)\end{array}$ \\
\hline $\begin{array}{l}\text { Asset Turnover } \\
\text { Ratio } \\
\end{array}$ & $\begin{array}{c}-50.36653 \\
(-1.46) \\
\end{array}$ & $\begin{array}{c}-50.36653 \\
(-0.92) \\
\end{array}$ & $\begin{array}{l}-270.7563 \\
(-2.52) * * \\
\end{array}$ & $\begin{array}{c}-41.41845 \\
(-0.94) \\
\end{array}$ & $\begin{array}{c}-41.41845 \\
(-0.63) \\
\end{array}$ \\
\hline \begin{tabular}{|l|} 
Operating \\
Expense Ratio \\
\end{tabular} & $\begin{array}{c}4.311229 \\
(1.53) \\
\end{array}$ & $\begin{array}{c}4.311229 \\
(0.67) \\
\end{array}$ & $\begin{array}{l}-24.36095 \\
(-1.99) * * \\
\end{array}$ & $\begin{array}{c}-0.5690485 \\
(-0.17) \\
\end{array}$ & $\begin{array}{c}-0.5690485 \\
(-0.07) \\
\end{array}$ \\
\hline SGA Ratio & $\begin{array}{c}0.4773626 \\
(0.21) \\
\end{array}$ & $\begin{array}{c}0.4773626 \\
(0.18)\end{array}$ & $\begin{array}{c}2.530291 \\
(0.91)\end{array}$ & $\begin{array}{c}1.959889 \\
(1.29)\end{array}$ & $\begin{array}{c}1.959889 \\
(0.53)\end{array}$ \\
\hline NOIVOL1 & $\begin{array}{l}-16.88015 \\
(-2.85)^{* * * *} \\
\end{array}$ & $\begin{array}{c}-16.88015 \\
(-1.55) \\
\end{array}$ & $\begin{array}{l}-143.063 \\
(-2.19)^{* *}\end{array}$ & $\begin{array}{c}.672065 \\
(0.49) \\
\end{array}$ & $\begin{array}{c}4.672065 \\
(0.22) \\
\end{array}$ \\
\hline NIVOL1 & $\begin{array}{c}1.877409 \\
(1.80)^{*} \\
\end{array}$ & $\begin{array}{c}1.877409 \\
(1.18) \\
\end{array}$ & $\begin{array}{c}8.660591 \\
(1.67)^{*} \\
\end{array}$ & $\begin{array}{c}-1.752239 \\
(-1.30) \\
\end{array}$ & $\begin{array}{c}-1.752239 \\
(-0.68) \\
\end{array}$ \\
\hline Size & $\begin{array}{c}-2.244587 \\
(-0.51) \\
\end{array}$ & $\begin{array}{c}-2.244587 \\
(-0.33) \\
\end{array}$ & $\begin{array}{l}-31.73766 \\
(-1.96) * * \\
\end{array}$ & $\begin{array}{c}1.951426 \\
(0.47) \\
\end{array}$ & $\begin{array}{c}1.951426 \\
(0.42) \\
\end{array}$ \\
\hline Age & $\begin{array}{c}0.0634363 \\
(0.37) \\
\end{array}$ & $\begin{array}{c}0.0634363 \\
(0.23)\end{array}$ & $\begin{array}{c}-0.5754502 \\
(-1.32)\end{array}$ & $\begin{array}{c}-0.0995373 \\
(-0.45)\end{array}$ & $\begin{array}{c}-0.0995373 \\
(-0.67)\end{array}$ \\
\hline Growth & $\begin{array}{l}-3.490572 \\
(-2.04)^{* *} \\
\end{array}$ & $\begin{array}{c}-3.490572 \\
(-1.44) \\
\end{array}$ & $\begin{array}{c}-0.4458783 \\
(-0.16)\end{array}$ & $\begin{array}{l}-4.623749 \\
(-4.78) * * * \\
\end{array}$ & $\begin{array}{l}-4.623749 \\
(-6.24) * * * \\
\end{array}$ \\
\hline Leverage & $\begin{array}{l}-15.26692 \\
(-3.36)^{* * * *} \\
\end{array}$ & $\begin{array}{c}-15.26692 \\
(-1.73)^{*} \\
\end{array}$ & $\begin{array}{c}15.12774 \\
(1.29) \\
\end{array}$ & $\begin{array}{c}3.187229 \\
(0.30) \\
\end{array}$ & $\begin{array}{c}3.187229 \\
(0.13) \\
\end{array}$ \\
\hline Divpay ratio & $\begin{array}{c}0.0366936 \\
(0.27) \\
\end{array}$ & $\begin{array}{c}0.0366936 \\
(0.13) \\
\end{array}$ & $\begin{array}{c}0.1460653 \\
(0.99) \\
\end{array}$ & $\begin{array}{c}-0.2650906 \\
(-0.54)\end{array}$ & $\begin{array}{c}-0.2650906 \\
(-0.34) \\
\end{array}$ \\
\hline Profitability & $\begin{array}{c}103.2204 \\
(82.95) * * *\end{array}$ & $\begin{array}{c}103.2204 \\
(41.67)^{* * * *}\end{array}$ & $\begin{array}{l}100.6358 \\
(7.72)^{* * * *}\end{array}$ & $\begin{array}{c}105.1719 \\
(65.46)^{* * *}\end{array}$ & $\begin{array}{c}105.1719 \\
(30.30)^{* * * *}\end{array}$ \\
\hline Constant & $\begin{array}{c}24.76311 \\
(0.89) \\
\end{array}$ & $\begin{array}{c}24.76311 \\
(0.58) \\
\end{array}$ & $\begin{array}{l}243.3632 \\
(2.14)^{* * *}\end{array}$ & $\begin{array}{c}-2.90929 \\
(-0.10) \\
\end{array}$ & $\begin{array}{c}-2.90929 \\
(-0.08) \\
\end{array}$ \\
\hline \begin{tabular}{|l|} 
Sargan Test \\
\end{tabular} & $\begin{array}{c}3.693257 \\
{[1.0000]} \\
\end{array}$ & & $\begin{array}{c}2.252914 \\
{[1.0000]}\end{array}$ & $\begin{array}{c}9.197519 \\
{[0.9998]} \\
\end{array}$ & \\
\hline $\operatorname{AR}(1)$ & $\begin{array}{c}-1.9187 \\
{[0.0550]^{*}}\end{array}$ & $\begin{array}{c}-1.8111 \\
{[0.0701]^{*}}\end{array}$ & $\begin{array}{c}1.3218 \\
{[0.1862]}\end{array}$ & $\begin{array}{c}-1.686 \\
{[0.0918]^{*}} \\
\end{array}$ & $\begin{array}{c}-1.5613 \\
{[0.1184]} \\
\end{array}$ \\
\hline $\operatorname{AR}(2)$ & $\begin{array}{c}-0.14142 \\
{[0.8875]}\end{array}$ & $\begin{array}{l}-0.11896 \\
{[0.9053]}\end{array}$ & $\begin{array}{c}1.6081 \\
{[0.1078]}\end{array}$ & $\begin{array}{c}1.0728 \\
{[0.2834]}\end{array}$ & $\begin{array}{c}0.58817 \\
{[0.5564]}\end{array}$ \\
\hline $\begin{array}{l}\text { No of } \\
\text { Observations }\end{array}$ & 80 & 80 & 61 & 85 & 85 \\
\hline $\begin{array}{l}\text { No of } \\
\text { Instruments }\end{array}$ & 45 & 45 & 41 & 43 & 43 \\
\hline
\end{tabular}

Notes: The z-statistics are in parentheses and p-values are in squared brackets. The ${ }^{* * *},{ }^{* *}$, and * indicate significance at $1 \%, 5 \%$ and $10 \%$ level respectively.

4.8 The Analysis of Free Cash Flow and Agency Costs on Tobin $Q$ for the REITs in Asia 
The results in Table 4.8 shows that only Model 1, Model 2, Model 5 and Model 6 passed all the Sargan test and $A R(1)$ and $A R(2)$ tests respectively. The Model 3 and Model 4 failed to pass the $A R(2)$ test respectively.

The results in Table 4.8 show that free cash flow is not significant to Tobin $q$ at all in all the four models respectively.

The lagged Tobin $Q$ is significantly positive to Tobin $Q$. The lagged Tobin asserts a significantly positive relationship on Tobin $Q$ and it is dynamic in the model.

Besides that, asset turnover ratio and net income volatility 1 (NIVOL1) are significantly negative to Tobin $q$ in Model 1 respectively. These are the agency costs in REITs that would devalue the REITs. Thus, there are in the opposite direction of Tobin q.

However, interestingly, the net operating income volatility 1 (NOIVOL1) is significantly positive at $10 \%$ level to Tobin $q$ in Model 1 . This highlights the complexity of the capital market whereby the REIT unitholders also emphasize that net operating income is necessary to uphold the value of the REITs in Asia. The divergence from the expected net operating income would be detrimental to value of the REITs in Asia. This is consistent with Chen et al. (2012).

The growth is significantly positive at $1 \%$ level to Tobin $q$ in all the four models respectively. The results are consistent with the strand of literatures whereby growth is positively related to the value of firm, Tobin $q$. The growth enhances the value of REITs in Asia. Thus, it is in the same direction of Tobin q.

In addition, the age is significantly positive to Tobin $q$ in Model 1 and Model 5 respectively. This indicates that the older REITs in Asia have more competitive edge in enhancing the value of the REITs.

Nonetheless, size and profitability are significantly negative to Tobin q in Model 5 and Model 1 respectively.

Table 4.8 The Results of Two-Step Different GMM and Two-Step Different GMM with Robust Standard Error of Free Cash Flow and Agency Costs on Tobin Q for the REITs in Asia. 


\begin{tabular}{|c|c|c|c|c|c|c|}
\hline Items & $\begin{array}{c}\text { Two-Step } \\
\text { Diff GMM } \\
\\
\text { Model } 1 \\
\text { TOBIN Q } \\
\end{array}$ & \begin{tabular}{|c|} 
Two-Step \\
Diff GMM with \\
Robust SE \\
Model 2 \\
TOBIN Q \\
\end{tabular} & $\begin{array}{c}\text { Two-Step } \\
\text { Diff GMM } \\
\\
\text { Model } 3 \\
\text { TOBIN Q } \\
\end{array}$ & \begin{tabular}{|c|} 
Two-Step \\
Diff GMM with \\
Robust SE \\
Model 4 \\
TOBIN Q \\
\end{tabular} & $\begin{array}{c}\text { Two-Step } \\
\text { Diff GMM } \\
\\
\text { Model } 5 \\
\text { TOBIN Q } \\
\end{array}$ & \begin{tabular}{|c|} 
Two-Step \\
Diff GMM with \\
Robust SE \\
Model 6 \\
TOBIN Q \\
\end{tabular} \\
\hline Lagged 1 & $\begin{array}{c}0.0761267 \\
(2.38)^{* *}\end{array}$ & $\begin{array}{c}0.0761267 \\
(1.00)\end{array}$ & $\begin{array}{c}0.0302033 \\
(1.05)\end{array}$ & $\begin{array}{c}0.0302033 \\
(0.66)\end{array}$ & $\begin{array}{c}0.0074446 \\
(0.25)\end{array}$ & $\begin{array}{c}0.0074446 \\
(0.10)\end{array}$ \\
\hline FCF1 & $\begin{array}{c}-0.0394682 \\
(-0.13)\end{array}$ & $\begin{array}{c}-0.0394682 \\
(-0.08)\end{array}$ & & & & \\
\hline FCF2 & & & $\begin{array}{c}2.066546 \\
(1.01)\end{array}$ & $\begin{array}{c}2.066546 \\
(0.81)\end{array}$ & & \\
\hline FCF3 & & & & & $\begin{array}{c}-2.63 \mathrm{E}-08 \\
(-1.60)\end{array}$ & $\begin{array}{c}-2.63 \mathrm{E}-08 \\
(-0.68)\end{array}$ \\
\hline $\begin{array}{l}\text { Asset Turnover } \\
\text { Ratio }\end{array}$ & $\begin{array}{l}-1.247103 \\
(-2.71)^{* * *}\end{array}$ & $\begin{array}{c}-1.247103 \\
(-0.65)\end{array}$ & $\begin{array}{c}-0.4629657 \\
(-0.62)\end{array}$ & $\begin{array}{c}-0.4629657 \\
(-0.55)\end{array}$ & $\begin{array}{c}-0.4553138 \\
(-1.30)\end{array}$ & $\begin{array}{c}-0.4553138 \\
(-0.36)\end{array}$ \\
\hline \begin{tabular}{|l|} 
Operating \\
Expense Ratio
\end{tabular} & $\begin{array}{c}-0.0695495 \\
(-0.93)\end{array}$ & $\begin{array}{c}-0.0695495 \\
(-0.46)\end{array}$ & $\begin{array}{c}-0.1656911 \\
(-1.92)^{*}\end{array}$ & $\begin{array}{c}-0.1656911 \\
(-2.34)^{* *}\end{array}$ & $\begin{array}{c}-0.0764902 \\
(-0.81)\end{array}$ & $\begin{array}{c}-0.0764902 \\
(-0.27)\end{array}$ \\
\hline SGA Ratio & $\begin{array}{c}-0.0652911 \\
(-1.63)\end{array}$ & $\begin{array}{c}-0.0652911 \\
(-0.44)\end{array}$ & $\begin{array}{c}0.0033234 \\
(0.09)\end{array}$ & $\begin{array}{c}0.0033234 \\
(0.11)\end{array}$ & $\begin{array}{c}-0.0574791 \\
(-0.78)\end{array}$ & $\begin{array}{c}-0.0574791 \\
(-0.47)\end{array}$ \\
\hline NOIVOL1 & $\begin{array}{c}0.7040326 \\
(1.80)^{*}\end{array}$ & $\begin{array}{c}0.7040326 \\
(0.89)\end{array}$ & $\begin{array}{c}0.3671755 \\
(0.55)\end{array}$ & $\begin{array}{c}0.3671755 \\
(0.33)\end{array}$ & $\begin{array}{c}0.0150716 \\
(0.35)\end{array}$ & $\begin{array}{c}0.0150716 \\
(0.04)\end{array}$ \\
\hline NIVOL1 & $\begin{array}{c}-0.1513689 \\
(-2.06)^{* *}\end{array}$ & $\begin{array}{c}-0.1513689 \\
(-0.96)\end{array}$ & $\begin{array}{c}-0.0799202 \\
(-0.89)\end{array}$ & $\begin{array}{c}-0.0799202 \\
(-0.55)\end{array}$ & $\begin{array}{c}-0.0133152 \\
(-1.46)\end{array}$ & $\begin{array}{c}-0.0133152 \\
(-0.30)\end{array}$ \\
\hline Size & $\begin{array}{c}-0.1426774 \\
(-1.44)\end{array}$ & $\begin{array}{c}-0.1426774 \\
(-0.58)\end{array}$ & $\begin{array}{c}-0.1102791 \\
(-1.02)\end{array}$ & $\begin{array}{c}-0.1102791 \\
(-0.66)\end{array}$ & $\begin{array}{c}-0.2010777 \\
(-1.98)^{* *}\end{array}$ & $\begin{array}{c}-0.2010777 \\
(-0.94)\end{array}$ \\
\hline Age & $\begin{array}{l}0.0122959 \\
(2.75)^{* * * *}\end{array}$ & $\begin{array}{c}0.0122959 \\
(1.02)\end{array}$ & $\begin{array}{c}0.0074649 \\
(1.91)^{*}\end{array}$ & $\begin{array}{c}0.0074649 \\
(1.07)\end{array}$ & $\begin{array}{c}0.0138992 \\
(2.06) * *\end{array}$ & $\begin{array}{c}0.0138992 \\
(1.17)\end{array}$ \\
\hline Growth & $\begin{array}{c}1.018151 \\
(101.83)^{* * * *}\end{array}$ & $\begin{array}{c}1.018151 \\
(15.07) * * *\end{array}$ & $\begin{array}{l}0.9983419 \\
(27.05)^{* * * *}\end{array}$ & $\begin{array}{l}0.9983419 \\
(29.17) * * *\end{array}$ & $\begin{array}{c}1.011719 \\
(48.06)^{* * * *}\end{array}$ & $\begin{array}{c}1.011719 \\
(18.37) * * *\end{array}$ \\
\hline Leverage & $\begin{array}{c}-0.2651107 \\
(-1.28)\end{array}$ & $\begin{array}{c}-0.2651107 \\
(-0.40)\end{array}$ & $\begin{array}{c}0.1759441 \\
(0.43)\end{array}$ & $\begin{array}{c}0.1759441 \\
(0.46)\end{array}$ & $\begin{array}{c}-0.0348452 \\
(-0.50)\end{array}$ & $\begin{array}{c}-0.0348452 \\
(-0.12)\end{array}$ \\
\hline Divpay ratio & $\begin{array}{c}0.003155 \\
(1.59)\end{array}$ & $\begin{array}{c}0.003155 \\
(0.41)\end{array}$ & $\begin{array}{c}0.0141201 \\
(1.08)\end{array}$ & $\begin{array}{c}0.0141201 \\
(0.87)\end{array}$ & $\begin{array}{c}-0.0003517 \\
(-0.12)\end{array}$ & $\begin{array}{c}-0.0003517 \\
(-0.06)\end{array}$ \\
\hline Profitability & $\begin{array}{c}-0.1993538 \\
(-1.72)^{*}\end{array}$ & $\begin{array}{c}-0.1993538 \\
(-0.62)\end{array}$ & $\begin{array}{c}-1.443588 \\
(-1.05)\end{array}$ & $\begin{array}{c}-1.443588 \\
(-0.86)\end{array}$ & $\begin{array}{c}-0.0037814 \\
(-0.20)\end{array}$ & $\begin{array}{c}-0.0037814 \\
(-0.09)\end{array}$ \\
\hline $\begin{array}{l}\text { Market } \\
\text { Return }\end{array}$ & $\begin{array}{c}0.0068626 \\
(1.22)\end{array}$ & $\begin{array}{c}0.0068626 \\
(0.44)\end{array}$ & $\begin{array}{c}-0.003028 \\
(-0.30)\end{array}$ & $\begin{array}{c}-0.003028 \\
(-0.19)\end{array}$ & $\begin{array}{c}-0.0033728 \\
(-0.42)\end{array}$ & $\begin{array}{c}-0.0033728 \\
(-0.13)\end{array}$ \\
\hline Constant & $\begin{array}{c}0.8990292 \\
(1.60)\end{array}$ & $\begin{array}{c}0.8990292 \\
(0.59)\end{array}$ & $\begin{array}{c}0.6125118 \\
(0.80)\end{array}$ & $\begin{array}{c}0.6125118 \\
(0.52)\end{array}$ & $\begin{array}{l}1.170796 \\
(2.14) * *\end{array}$ & $\begin{array}{c}1.170796 \\
(0.93)\end{array}$ \\
\hline Sargan Test & $\begin{array}{l}8.210824 \\
{[1.0000]} \\
\end{array}$ & & $\begin{array}{l}2.05764 \\
{[1.0000]}\end{array}$ & & $\begin{array}{l}12.99841 \\
{[0.9970]}\end{array}$ & \\
\hline $\operatorname{AR}(\mathbf{1})$ & $\begin{array}{l}-0.15891 \\
{[0.8737]}\end{array}$ & $\begin{array}{l}-0.10251 \\
{[0.9183]}\end{array}$ & $\begin{array}{l}-0.90434 \\
{[0.3658]}\end{array}$ & $\begin{array}{l}-0.8547 \\
{[0.3927]}\end{array}$ & $\begin{array}{c}-1.1136 \\
{[0.2655]}\end{array}$ & $\begin{array}{c}-0.5948 \\
{[0.5520]}\end{array}$ \\
\hline $\mathbf{A R}(2)$ & $\begin{array}{l}-1.1938 \\
{[0.2325]}\end{array}$ & $\begin{array}{l}-0.92895 \\
{[0.3529]}\end{array}$ & $\begin{array}{c}-1.8103 \\
{[0.0703]^{*}}\end{array}$ & $\begin{array}{c}-1.8048 \\
{[0.0711]^{*}}\end{array}$ & $\begin{array}{l}-1.3948 \\
{[0.1631]}\end{array}$ & $\begin{array}{l}-1.1456 \\
{[0.2519]}\end{array}$ \\
\hline \begin{tabular}{|l|} 
No of \\
Observations
\end{tabular} & 88 & 88 & 66 & 66 & 94 & 94 \\
\hline \begin{tabular}{|l|} 
No of \\
Instruments
\end{tabular} & 47 & 47 & 43 & 43 & 45 & 45 \\
\hline
\end{tabular}

Notes: The z-statistics are in parentheses and $p$-values are in squared brackets. The ${ }^{* * *},{ }^{* *}$, and ${ }^{*}$ indicate significance at $1 \%, 5 \%$ and $10 \%$ level respectively.

\subsection{The Analysis of Free Cash Flow and Agency Costs on REIT Return for the REITs in Asia}


The results in Table 4.9 show that all models passed all the Sargan test and $A R(1)$ and $A R(2)$ tests respectively.

The results in Table 4.9 show that free cash flow is not significant to REIT return at all in all models respectively.

However, the lagged REIT return is significantly negative to REIT return in Model 1, Model 3, Model 4 and Model 5 respectively and it is dynamic in the Model 1, Model 3, Model 4 and Model 5 respectively.

The net income volatility 1 (NIVOL1) is significantly negative to REIT return in Model 3. The net income volatility 1 (NIVOL1) is the agency cost in REITs that would be detrimental to REIT return. Thus, it is in the opposite direction of REIT return.

The growth is significantly positive to REIT return in all models respectively except Model 6. The growth enhances the return of REITs in Asia. Thus, it is in the same direction of REIT return.

In addition, the age is significantly positive to REIT return in all models respectively except Model 4 and Model 6 . This indicates that the older REITs in Asia have more competitive edge in increasing the REIT return.

Besides that, market return is significantly positive to REIT return in all models respectively. Market return accelerates the REIT return in Asia. Hence, it is in the same direction of REIT return. This is consistent with the strand of literatures that market return is always positively related to the return.

Nonetheless, size is significantly negative to REIT return in Model 1.

Table 4.9 The Results of Two-Step Different GMM and Two-Step Different GMM with Robust Standard Error of Free Cash Flow and Agency Costs on REIT Return for the REITs in Asia. 


\begin{tabular}{|c|c|c|c|c|c|c|}
\hline Items & $\begin{array}{c}\text { Two-Step } \\
\text { Diff GMM } \\
\text { Model } 1 \\
\text { REIT RETURN } \\
\end{array}$ & $\begin{array}{c}\text { Two-Step } \\
\text { Diff GMM with } \\
\text { Robust SE } \\
\text { Model } 2 \\
\text { REIT RETURN }\end{array}$ & $\begin{array}{c}\text { Two-Step } \\
\text { Diff GMM } \\
\text { Model } 3 \\
\text { REIT RETURN } \\
\end{array}$ & $\begin{array}{c}\text { Two-Step } \\
\text { Diff GMM with } \\
\text { Robust SE } \\
\text { Model } 4 \\
\text { REIT RETURN } \\
\end{array}$ & $\begin{array}{c}\text { Two-Step } \\
\text { Diff GMM } \\
\text { Model } 5 \\
\text { REIT RETURN } \\
\end{array}$ & $\begin{array}{c}\text { Two-Step } \\
\text { Diff GMM with } \\
\text { Robust SE } \\
\text { Model } 6 \\
\text { REIT RETURN }\end{array}$ \\
\hline Lagged 1 & $\begin{array}{l}-0.113998 \\
(-2.34)^{* *}\end{array}$ & $\begin{array}{c}-0.113998 \\
(-1.48) \\
\end{array}$ & $\begin{array}{l}-0.1777892 \\
(-2.78) * * *\end{array}$ & $\begin{array}{c}-0.1777892 \\
(-1.81)^{*}\end{array}$ & $\begin{array}{l}-0.1113121 \\
(-3.13) * * *\end{array}$ & $\begin{array}{c}-0.1113121 \\
(-1.56)\end{array}$ \\
\hline FCF1 & $\begin{array}{c}-4.497449 \\
(-0.95) \\
\end{array}$ & $\begin{array}{c}-4.497449 \\
(-0.75) \\
\end{array}$ & & & & \\
\hline FCF2 & & & $\begin{array}{c}8.619044 \\
(0.84)\end{array}$ & $\begin{array}{c}8.619044 \\
(0.66)\end{array}$ & & \\
\hline FCF3 & & & & & $\begin{array}{c}-1.79 \mathrm{E}-07 \\
(-0.59) \\
\end{array}$ & $\begin{array}{c}-1.79 \mathrm{E}-07 \\
(-0.27) \\
\end{array}$ \\
\hline $\begin{array}{l}\text { Asset Turnover } \\
\text { Ratio } \\
\end{array}$ & $\begin{array}{c}-3.224557 \\
(-0.57) \\
\end{array}$ & $\begin{array}{c}-3.224557 \\
(-0.33) \\
\end{array}$ & $\begin{array}{c}2.187819 \\
(0.16) \\
\end{array}$ & $\begin{array}{c}2.187819 \\
(0.12) \\
\end{array}$ & $\begin{array}{c}3.340523 \\
(0.53) \\
\end{array}$ & $\begin{array}{c}3.340523 \\
(0.34) \\
\end{array}$ \\
\hline \begin{tabular}{|l|} 
Operating \\
Expense Ratio \\
\end{tabular} & $\begin{array}{c}-0.5409539 \\
(-0.71) \\
\end{array}$ & $\begin{array}{c}-0.5409539 \\
(-0.42) \\
\end{array}$ & $\begin{array}{c}-2.181398 \\
(-0.87) \\
\end{array}$ & $\begin{array}{c}-2.181398 \\
(-0.66) \\
\end{array}$ & $\begin{array}{c}-1.338955 \\
(-1.26) \\
\end{array}$ & $\begin{array}{c}-1.338955 \\
(-0.62) \\
\end{array}$ \\
\hline SGA Ratio & $\begin{array}{c}-0.0649702 \\
(-0.16) \\
\end{array}$ & $\begin{array}{c}-0.0649702 \\
(-0.05) \\
\end{array}$ & $\begin{array}{c}-0.1024365 \\
(-0.25) \\
\end{array}$ & $\begin{array}{c}-0.1024365 \\
(-0.19) \\
\end{array}$ & $\begin{array}{c}0.3265676 \\
(0.39) \\
\end{array}$ & $\begin{array}{c}0.3265676 \\
(0.14) \\
\end{array}$ \\
\hline NOIVOL1 & $\begin{array}{c}1.726244 \\
(0.50) \\
\end{array}$ & $\begin{array}{c}1.726244 \\
(0.26) \\
\end{array}$ & $\begin{array}{c}24.50123 \\
(1.59) \\
\end{array}$ & $\begin{array}{c}24.50123 \\
(0.86) \\
\end{array}$ & $\begin{array}{c}5.682061 \\
(1.53) \\
\end{array}$ & $\begin{array}{c}5.682061 \\
(0.84) \\
\end{array}$ \\
\hline NIVOL1 & $\begin{array}{c}-0.4664968 \\
(-0.66) \\
\end{array}$ & $\begin{array}{c}-0.4664968 \\
(-0.36) \\
\end{array}$ & $\begin{array}{c}-3.762521 \\
(-1.80)^{*} \\
\end{array}$ & $\begin{array}{c}-3.762521 \\
(-0.98) \\
\end{array}$ & $\begin{array}{c}-1.136265 \\
(-1.48) \\
\end{array}$ & $\begin{array}{c}-1.136265 \\
(-0.89) \\
\end{array}$ \\
\hline Size & $\begin{array}{l}-1.529655 \\
(-2.10)^{* *} \\
\end{array}$ & $\begin{array}{c}-1.529655 \\
(-1.13) \\
\end{array}$ & $\begin{array}{c}-1.076387 \\
(-0.74) \\
\end{array}$ & $\begin{array}{c}-1.076387 \\
(-0.56) \\
\end{array}$ & $\begin{array}{c}-0.7303293 \\
(-0.96) \\
\end{array}$ & $\begin{array}{c}-0.7303293 \\
(-0.54) \\
\end{array}$ \\
\hline Age & $\begin{array}{l}0.1102015 \\
(2.82)^{* * * *}\end{array}$ & $\begin{array}{c}0.1102015 \\
(1.87)^{*}\end{array}$ & $\begin{array}{c}0.1509851 \\
(2.11)^{* *}\end{array}$ & $\begin{array}{c}0.1509851 \\
(0.76) \\
\end{array}$ & $\begin{array}{c}0.0860017 \\
(2.14)^{* *}\end{array}$ & $\begin{array}{c}0.0860017 \\
(1.24) \\
\end{array}$ \\
\hline Growth & $\begin{array}{l}1.165736 \\
(3.60)^{* * *}\end{array}$ & $\begin{array}{c}1.165736 \\
(1.94)^{*} \\
\end{array}$ & $\begin{array}{l}1.369911 \\
(3.80)^{* * *} \\
\end{array}$ & $\begin{array}{c}1.369911 \\
(1.65)^{*} \\
\end{array}$ & $\begin{array}{l}0.948883 \\
(3.04)^{* * *} \\
\end{array}$ & $\begin{array}{c}0.948883 \\
(1.53) \\
\end{array}$ \\
\hline Leverage & $\begin{array}{c}-0.0143927 \\
(-0.01) \\
\end{array}$ & $\begin{array}{c}-0.0143927 \\
(-0.01) \\
\end{array}$ & $\begin{array}{c}0.9158451 \\
(0.29) \\
\end{array}$ & $\begin{array}{c}0.9158451 \\
(0.10) \\
\end{array}$ & $\begin{array}{c}1.687781 \\
(1.26) \\
\end{array}$ & $\begin{array}{c}1.687781 \\
(0.78) \\
\end{array}$ \\
\hline Divpay ratio & $\begin{array}{c}0.0251351 \\
(0.46)\end{array}$ & $\begin{array}{c}0.0251351 \\
(0.30) \\
\end{array}$ & $\begin{array}{c}0.1329476 \\
(1.33) \\
\end{array}$ & $\begin{array}{c}0.1329476 \\
(0.87) \\
\end{array}$ & $\begin{array}{c}0.0506823 \\
(1.05) \\
\end{array}$ & $\begin{array}{c}0.0506823 \\
(0.55) \\
\end{array}$ \\
\hline Profitability & $\begin{array}{c}-0.6437169 \\
(-1.33) \\
\end{array}$ & $\begin{array}{c}-0.6437169 \\
(-0.77) \\
\end{array}$ & $\begin{array}{c}-5.813443 \\
(-0.92) \\
\end{array}$ & $\begin{array}{c}-5.813443 \\
(-0.71) \\
\end{array}$ & $\begin{array}{c}-0.0635056 \\
(-0.15) \\
\end{array}$ & $\begin{array}{c}-0.0635056 \\
(-0.10) \\
\end{array}$ \\
\hline $\begin{array}{l}\text { Market } \\
\text { Return } \\
\end{array}$ & $\begin{array}{c}0.8077942 \\
(8.37)^{* * *}\end{array}$ & $\begin{array}{c}0.8077942 \\
(6.05) * * *\end{array}$ & $\begin{array}{l}0.858161 \\
(8.50)^{* * * *}\end{array}$ & $\begin{array}{l}0.858161 \\
(5.37)^{* * *}\end{array}$ & $\begin{array}{l}0.8303118 \\
(10.53)^{* * * *}\end{array}$ & $\begin{array}{c}0.8303118 \\
(6.52)^{* * *}\end{array}$ \\
\hline Constant & $\begin{array}{c}8.444636 \\
(1.82)^{*} \\
\end{array}$ & $\begin{array}{c}8.444636 \\
(0.97) \\
\end{array}$ & $\begin{array}{c}4.822821 \\
(0.45) \\
\end{array}$ & $\begin{array}{c}.822821 \\
(0.34) \\
\end{array}$ & $\begin{array}{c}3.138437 \\
(0.64) \\
\end{array}$ & $\begin{array}{c}3.138437 \\
(0.36) \\
\end{array}$ \\
\hline Sargan Test & $\begin{array}{c}8.755609 \\
{[1.0000]} \\
\end{array}$ & & $\begin{array}{c}5.281444 \\
{[1.0000]} \\
\end{array}$ & & $\begin{array}{c}9.852532 \\
{[0.9998]} \\
\end{array}$ & \\
\hline $\operatorname{AR}(1)$ & $\begin{array}{c}-1.8633 \\
{[0.0624]^{*}}\end{array}$ & $\begin{array}{c}-1.5508 \\
{[0.1210]} \\
\end{array}$ & $\begin{array}{l}-0.43691 \\
{[0.6622]} \\
\end{array}$ & $\begin{array}{c}-0.1929 \\
{[0.8470]} \\
\end{array}$ & $\begin{array}{l}-1.2205 \\
{[0.2223]} \\
\end{array}$ & $\begin{array}{c}-1.0231 \\
{[0.3063]} \\
\end{array}$ \\
\hline $\mathbf{A R}(2)$ & $\begin{array}{l}-0.71491 \\
{[0.4747]} \\
\end{array}$ & $\begin{array}{c}-0.5754 \\
{[0.5650]} \\
\end{array}$ & $\begin{array}{c}1.3459 \\
{[0.1783]} \\
\end{array}$ & $\begin{array}{l}0.77365 \\
{[0.4391]} \\
\end{array}$ & $\begin{array}{c}-0.3184 \\
{[0.7502]} \\
\end{array}$ & $\begin{array}{l}-0.28504 \\
{[0.7756]} \\
\end{array}$ \\
\hline $\begin{array}{l}\text { No of } \\
\text { Observations }\end{array}$ & 86 & 86 & 64 & 64 & 92 & 92 \\
\hline $\begin{array}{l}\text { No of } \\
\text { Instruments }\end{array}$ & 47 & 47 & 43 & 43 & 45 & 45 \\
\hline
\end{tabular}

Notes: The z-statistics are in parentheses and p-values are in squared brackets. The ${ }^{* * *},{ }^{* *}$, and ${ }^{*}$ indicate significance at $1 \%, 5 \%$ and $10 \%$ level respectively.

\subsection{CONCLUSIONS}

This study contributes to the limited literature on Asian REITs by examining the impacts of free cash flow and agency costs on the performance of REITs in Asia. This 
study employs GMM method which is more robust compared to previous studies that used pooled OLS and panel data method.

By using panel data of the four biggest market capitalization of Asian REITs, namely, Japan, Singapore, Hong Kong and Malaysia, firstly, this paper examines the impacts of free cash flow on agency costs. Secondly, this paper also assesses the impacts of these free cash flow and agency costs on the performance of REITs in Asia.

The findings indicate that there are significant impacts of free cash flow on agency costs such as on the asset turnover, operating expense ratio, selling, general and administrative expenses ratio (SGAR), net operating income volatility 1 (NOIVOL1) and net income volatility 1 (NIVOL1) respectively.

Interestingly, the results suggest that the free cash flow supports free cash flow theory in one hypothesis but it does not support free cash flow theory in another hypothesis. This shows that the free cash flow is minimal due to the dual directions of the free cash flow theory in the findings.

This also implies that the inherent risk associated with free cash flow is minimal in the REITs. This can be attributed to the "REIT effect" (Bauer, Eichholtz and Kok, 2010) whereby REITs payout $90 \%$ of its earning as distribution or dividend payout for tax exemption. The deviation from optimal free cash flow is minimum and less costly. Moreover, the REIT managers know how to gauge managerial optimism in REITs.

In addition, this study also indicates that free cash flow and agency costs exist and persist over time in Asian REITs even though REITs are in a highly regulated industry. The findings indicate that the agency costs such as asset turnover and operating expense ratio persist over time and affect the REIT performance, return on assets (ROA) and return on equity (ROE) negatively and respectively. Besides that, asset turnover and net income volatility (NIVOL1) have significant negative impacts on the REIT value. These agency costs discount the value of REITs in Asia. Moreover, the net income volatility (NIVOL1) decreases the REIT return. Hence, the agency costs persist and affect the performance of REITs in Asia.

The lagged individual agency cost such as lagged asset turnover, lagged SGA ratio, lagged NOIVOL1 and lagged NIVOL1 are significantly positive to its agency cost respectively. These lagged individual agency cost induces higher agency costs in REITs in Asia. There are dynamic and complex in the models.

These findings also imply that REIT managers face substantial costs when they wish to adjust to the equilibrium level of agency costs, whereby the optimum level is always dynamic and not constant over time and moves with the changes in the determinants of agency costs. These agency costs persist over time. The adjustment speed is dependent upon on the lagged individual agency cost. The adjustment speed is given by 1 minus the estimated coefficient of the lagged dependent variable.

This also implies that the REITs in Asia should consider internally managed REIT structure since the agency costs persist over time and there are always dynamic and not constant over time and moves with the changes in the determinants of agency costs. 
Moreover, the internally managed REIT structure provides empirical evidences that they outperform the externally managed REIT structure in the US REITs studies (Cannon and Vogt, 1995; Capozza and Seguin, 2000; Clayton and MacKinnon, 2000; Ambrose and Linneman, 2001).

Besides that, the findings depict that the Asian REITs possess their own unique characteristics. They differ in term of size, age and other aspects that may cause Asian REITs to vary differently. These include financial position, operation management, economy of scale and expertise in REIT management skills that may have different and significant impacts on the performance of REITs in Asia. The findings also show that the leverage, dividend payout ratio, growth and profitability can help to mitigate or induce agency costs in the REITs in Asia.

The findings also imply that the regulators in Asian REITs should enforce absolute stringent corporate governance rules and regulations in order to govern the existing inherent handicapped satellite structure of the externally managed REITs in Asia.

In addition, the Asian REITs regulators should plan to have REITs that are internally managed; and to provide expertise, education and training for the existing externally managed REITs to transform into internally managed REIT structure. This can be done through detailed planning and implementation of the project within a proper time frame.

For other countries in Asia such as India, China, Philippines, Indonesia and Pakistan, they should consider absolute stringent corporate governance rules and regulations on REITs and internally managed REIT structure in order to provide sufficient investor protection and to establish a more robust REIT market in these important emerging markets.

For REIT regulators in Asia, this empirical study helps to provide useful information for policy planning and formulation in REIT corporate governance; and to transform the inherent handicapped satellite structure of the externally managed REITs into internally managed REIT structure.

For REIT managers and practitioners, this empirical study also helps them to be more aware of the dynamism of free cash flow and agency costs in REITs; and alert them that these free cash flow and agency costs persist over time which can have significant impacts on the REIT performance, return on assets (ROA) and return on equity (ROE), REIT value and REIT return respectively in Asia. Thus, they could consider internalizing their REIT management structure.

As a whole, this empirical study contributes significant benefits to all levels of the REIT industry in Asia. Lastly, for future research, researchers can embark on research studies on issues that might determine the speed of adjustment towards the equilibrium level of agency costs in Asian REITs. 


\section{References}

Adjaoud, F. and Ben-Amar, W. (2010). Corporate Governance and Dividend Policy: Shareholders' Protection or Expropriation? Journal of Business Finance and Accounting, 37(5-6), 648-667. 
Al-Najjar, B. (2013). The Financial Determinants of Corporate Cash Holdings: Evidence from Some Emerging Markets. International Business Review, 22(1), 77-88.

Al-Najjar, B. and Belghitar, Y. (2011). Corporate Cash Holdings and Dividend Payments: Evidence from Simultaneous Analysis. Managerial and Decision Economics, 32(4), 231-241.

Ambrose, B. and Linneman, P. (2001). REIT Organizational Structure and Operating Characteristics. Journal of Real Estate Research, 21, 146-162.

Ang, J. S., Cole, R. A. and Lin, J. W. (2000). Agency Costs and Ownership Structure. Journal of Finance, 55(1), 81-106.

Baker, G. P. (1993). Growth, Corporate Policies, and Investment Opportunity Set. Journal of Accounting and Economics, 16(1-3), 161-165.

Bartram, S., Brown, P., How, J. and Verhoevan, P. (2008). Agency Conflicts and Corporate Payout Policies: A Global Study. Working Paper, University of Auckland.

Bauer, R., Eichholtz, P. M. A. and Kok, N. (2010). Corporate Governance and Performance: The REIT Effect. Real Estate Economics, 38(1), 1-29.

Brush, T. H., Philip, B. and Margaretha, H. (2000). The Free Cash Flow Hypothesis for Sales Growth and Firm Performance. Strategic Management Journal, 21, 455-472.

Cannon, S. E. and Vogt, S. C. (1995). REITs and Their Management: An Analysis of Organizational Structure, Performance, and Management Compensation. Journal of Real Estate Research, 10(3), 297-318.

Capozza, D. R. and Seguin, P. J. (2000). Debt, Agency, and Management Contracts in REITs: The External Advisor Puzzle. Journal of Real Estate Finance and Economics, 20(2), 91-116.

Chae, J., Kim, S. and Lee, E. J. (2009). How Corporate Governance Affects Payout Policy Under Agency Problems and External Financing Constraints. Journal of Banking and Finance, 33(11), 2093-2101.

Chang, S. C., Chen. S. S., Hsing, A. and Huang, C. W. (2007). Investment Opportunities, Free Cash Flow, and Stock Valuation Effects of Secured Debt Offerings. Review of Quantitative Finance and Accounting, 28(2), 123-145.

Chi, J. D. and Lee, D. S. (2010). The Conditional Nature of the Value of Corporate Governance. Journal of Banking and Finance, 34(2), 350-361.

Chen, S. S., Chou, R. K. and Chou, S. F. (2009). The Impact of Investment Opportunities and Free Cash Flow on Financial Liberalization: A Cross-Firm Analysis of Emerging Economies. Financial Management, 38(3), 543-566.

Chen, S. S. and Chen, I. J. (2012). Corporate Governance and Capital Allocations of Diversified Firms. Journal of Banking and Finance, 36 (2), 395-409. 
Chen, M. C., Wang, C. Y. and Shyu, S. D. (2012). Liquidity and the Future Stock Returns of the REIT Industry. Journal of Real Estate Finance and Economics, 45(3), 588-603.

Chou, W. H., Hardin III, W. G., Hill, M. D. and Kelly, G. W. (2013). Dividends, Values and Agency Costs in REITs. Journal of Real Estate Finance and Economics, 46(1), $91-114$.

Chung, R., Fung, S. and Hung, K. S. (2012). Institutional Investors and Firm Efficiency of Real Estate Investment Trusts. Journal of Real Estate Finance and Economics, 45(1), 171-211.

Clayton, J. and MacKinnon, G. (2000). Measuring and Explaining Changes in REIT Liquidity: Moving Beyond the Bid-Ask Spread. Real Estate Economics, 28(1), 89-115.

Cleary, S. (2006). International Corporate Investment and the Relationships Between Financial Constraint Measures. Journal of Banking and Finance, 30(5), 1559-1580.

Coad, A., Segarra, A. and Teruel, M. (2013). Like Milk or Wine: Does Firm Performance Improve With Age? Structural Change and Economic Dynamics, 24(1), 173-189.

DeAngelo, H., DeAngelo, L. and Stulz, R. (2006). Dividend Policy and the Earned/Contributed Capital Mix: A Test of the Life-cycle Theory. Journal of Financial Economics, 81(2), 227-254.

Denis, D. and Osobov, I. (2008). Why Do Firms Pay Dividends? International Evidence on the Determinants of Dividend Policy. Journal of Financial Economics, 89(1), 62-82.

Easterbrook, F. (1984). Two Agency-cost Explanations of Dividends. American Economic Review, 74(4), 650-659.

Feng, Z., Ghosh, C. and Sirmans, C. F. (2007). CEO Involvement in Director Selection: Implications for REIT Dividend Policy. Journal of Real Estate Finance and Economics, 35(4), 385-410.

Flannery, M. J. and Hankins, K. W. (2013). Estimating Dynamic Panel Models in Corporate Finance. Journal of Corporate Finance, 19, 1-19.

Fleming, G., Heaney, R. and McCosker, R. (2005). Agency Costs and Ownership Structure in Australia. Pacific-Basin Finance Journal, 13(1), 29-52.

Florackis, C. (2008). Agency Costs and Corporate Governance Mechanisms: Evidence for UK Firms. International Journal of Managerial Finance, 4(1), 37-59.

Florackis, C. and Ozkan, A. (2009). The Impact of Managerial Entrenchment on Agency Costs: An Empirical Investigation Using UK Panel Data. European Financial Management, 15(3), 497-528. 
Ghosh, C., Petrova, M. and Xiao, Y. (2012). Do REITs Use Cash Reserves Efficiently? Evidence From Corporate Acquisitions. Journal of International Money and Finance, 31(7), 1953-1970.

Ghosh, C. and Sirman, C. F. (2006). Do Managerial Motives Impact Dividend Decisions in REITs. Journal of Real Estate Finance and Economic, 32(3), 327-355.

Gregory, A. (2005). The Long Run Abnormal Performance of UK Acquirers and the Free Cash Flow Hypothesis. Journal of Business Finance and Accounting, 32(5-6), 777-814.

Grossman, S. and Hart, O. D. (1982). Corporate Financial Structure and Managerial Incentives. In: McCall, J. (Ed), The Economics of Information and Uncertainty. University of Chicago Press, Chicago, IL, pp 107-140.

Gul, F. A. (1999). Growth Opportunities, Capital Structure and Dividend Policies in Japan. Journal of Corporate, 5(2), 141-168.

Gul, F. A. and Tsui, J. S. L. (1997). A Test of the Free Cash Flow and Debt Monitoring Hypotheses: Evidence From Auditing Pricing. Journal of Accounting and Economics, 24(2), 219-237.

Gul, F. A. and Tsui, J. S. L. (2001). Free Cash Flow, Debt Monitoring and Audit Pricing: Further Evidence on the Role of Director Equity Ownership. Journal of Practice and Theory, 20(2), 71-84.

Hardin III, W. G., Highfield, M. J., Hill, M. D. and Kelly, G. W. (2009). The Determinants of REIT Cash Holdings. Journal of Real Estate Finance and Economics, 39(1), 39-57.

He, W. (2012). Agency Problems, Product Market Competition and Dividend Policies in Japan. Accounting and Finance, 52(3), 873-901.

Henry, D. (2010). Agency Costs, Ownership Structure and Corporate Governance Compliance: A Private Contracting Perspective. Pacific-Basin Finance Journal, 18(1), 24-46.

Hijazi, B. M. and Conover, J. A. (2011). Empirical Performance of Accounting Measures of Direct Agency Costs. Reseach in Finance, 27, 223-272.

Hutchinson, M. and Gul, F. A. (2004). Investment Opportunities Set, Corporate Governance Practices and Firm Performance. Journal of Corporate Finance, 10(4), 595-614.

Iskandar, T. M., Bukit, R. B. and Sanusi, Z. M. (2012). The Moderating Effect of Ownership Structure on the Relationship Between Free Cash Flow and Asset Utilisation. Asian Academy of Management Journal of Accounting and Finance, 8(1), 69-89. 
Jirapon, P., Kim, J. C. and Kim, Y. S. (2011). Dividend Payouts and Corporate Governance Quality: An Empirical Investigation. The Financial Review, 46(2), 251279.

Jelinek, K. and Stuerke, P. S. (2009). The Nonlinear Relation Between Agency Costs and Managerial Equity Ownership. International Journal of Managerial Finance, 5(2), 156-178.

Jensen, M. C. (1986). Agency Cost of Free Cash Flow, Corporate Finance, and Takeovers. American Economic Review, 76(2), 323-329.

John, K. and Knyazeka, A. (2006). Payout Policy, Agency Conflicts and Corporate Governance. Working Paper, New York University.

John, K., Knyazeva, A. and Knyazeva, D. (2011). Does Geography Matter? Firm Location and Corporate Payout Policy. Journal of Financial Economics, 101(3), 533551.

Kim, C., Mauer, D. and Sherman, A. (1998). The Determinants of Corporate Liquidity: Theory and Evidence. Journal of Financial and Quantitative Analysis, 33(3), 335-359.

La Porta, R., Lopez-De-Silanes, F., Shleifer, A. and Vishny, R. (2000). Agency Problems and Dividend Policies Around the World. The Journal of Finance, 55(1), 133.

Lang, L. H. P., Stulz, R. M. and Walking, R. A. (1991). A Test of the Free Cash Flow Hypothesis. The Case of Bidder Returns. Journal of Financial Economics, 29(2), 315335.

Lecomte, P. and Ooi, J. T. L. (2013). Corporate Governance and Performance of Externally Managed Singapore Reits. Journal of Real Estate Finance and Economics, 46(4), 664-684.

Lee, C. F., Gupta, M. C., Chen, H. Y. and Lee, A. C. (2011). Optimal Payout Ratio Under Uncertainty and the Flexibility Hypothesis: Theory and Empirical Evidence. Journal of Corporate Finance, 17(3), 483-501.

Lehn, K. and Poulsen, A. (1989). Free Cash Flow and Stockholder Gains in Going Private Transactions. Journal of Finance, 44(3), 771-787.

Li, K. K. (2008). Expected Holding of Cash, Future Performance and Stock Return. Working Paper, University of California at Berkeley.

McKnight, P. J. and Weir, C. (2009). Agency Costs, Corporate Governance Mechanisms and Ownership Structure in Large UK Publicly Quoted Companies: A Panel Data Analysis. The Quarterly Review of Economics and Finance, 49(2), 139158.

Myers, S. C. (1977). The Determinants of Corporate Borrowing. Journal of Financial Economics, 5(2), 147-175. 
Myers, S. and Majluf, N. (1984). Corporate Financing and Investment Decisions When Firms Have Information That Investors Do Not Have. Journal of Financial Economics, 13(2), 187-221.

Newell, G. (2012). The Investment Characteristics and Benefits of Asian REITs for Retail Investors. Asia Pacific Real Estate Association Limited, Singapore.

Noguera, M. (2012). The Impact of the Sarbanes-Oxley Act on the Structure of REIT Boards of Directors. Journal of Real Estate Finance and Economics, 45(4), 869-887.

Oler, D. and Picconi, M. (2010). Implications of Insufficient and Excess Cash for Future Performance. Working Paper, Texas Tech University.

Ooi, J. T. L. and Liow, K. H. (2004). Risk-adjusted Performance of Real Estate Stocks: Evidence from Developing Market. Journal of Real Estate Research, 26(4), 371-395.

Opler, T., Pinkowitz, L., Stulz, R. and Williamson, R. (1999). The Determinants and Implications of Corporate Cash Holdings. Journal of Financial Economics, 52(1), 3-46.

Oprea, R. (2008). Free Cash Flow and Takeover Threats: An Experimental Study. Southern Economic Journal, 75(2), 351-366.

Richardson, S. (2006). Over-investment of Free Cash Flow. Review of Accounting Studies, 11(2-3), 159-189.

Rozeff, M. (1982). Growth, Beta and Agency Cost as Determinants of Dividend Payout Ratios. Journal of Financial Research, 5(3), 249-259.

Singh, M. and Davidson III, W. N. (2003). Agency Costs, Ownership Structure and Corporate Governance Mechanisms. Journal of Banking and Finance, 27(5), 793-816.

Smith, C. and Watts, R. (1992). The Investment Opportunity Set and Corporate Financing, Dividend and Compensation Policies. Journal of Financial Economics, 32(3), 263-292.

Wang, G. Y. (2010). The Impacts of Free Cash Flows and Agency Costs on Firm Performance. Journal of Service Science and Management, 3(4), 408-418.

Williams, J. (1987). Perquisites, Risk, and Capital Structure. Journal of Finance, 42(1), 29-48.

Wintoki, M. B., Linck, J. S. and Netter, J. M. (2012). Endogeneity and the Dynamics of Internal Corporate Governance. Journal of Financial Economics, 105(3), 581-606.

Wu, L. (2004). The Impact of Ownership Structure on Debt Financing of Japanese Firms with the Agency Cost of Free Cash Flow. Working Paper, City University of Hong Kong. 
Zwiebel, J. (1996). Dynamic Capital Structure Under Managerial Entrenchment. American Economic Review, 86(5), 1197-1215. 